\title{
Natural Products Modulate Cell Apoptosis: A Promising Way for the Treatment of Ulcerative Colitis
}

\section{OPEN ACCESS}

Edited by:

Xin Luan,

Shanghai University of Traditional Chinese Medicine, China

Reviewed by:

Songqi Tang,

Hainan Medical University, China

Fengjie Zheng,

Beijing University of Chinese Medicine,

China

*Correspondence:

Yi LiU

tcmly@163.com

tORCID:

Chenhao Liu

orcid.org/0000-0003-2563-2760

Yiwei Zeng

orcid.org/0000-0002-7042-2503

FThese authors have contributed equally to this work

Specialty section:

This article was submitted to Ethnopharmacology,

a section of the journal

Frontiers in Pharmacology

Received: 31 October 2021

Accepted: 10 January 2022

Published: 31 January 2022

Citation:

Liu C, Zeng $Y$, Wen $Y$, Huang $X$ and

Liu $Y$ (2022) Natural Products

Modulate Cell Apoptosis: A Promising

Way for the Treatment of

Ulcerative Colitis.

Front. Pharmacol. 13:806148.

doi: 10.3389/fphar.2022.806148

\author{
Chenhao Liu ${ }^{1+\neq}$, Yiwei Zeng ${ }^{2+\neq}$, Yulong Wen ${ }^{1}$, Xinggui Huang ${ }^{1}$ and Yi Liu ${ }^{1 *}$ \\ ${ }^{1}$ School of Basic Medical Sciences, Chengdu University of Traditional Chinese Medicine, Chengdu, China, ${ }^{2}$ School of \\ Acupuncture-Moxibustion and Tuina, Chengdu University of Traditional Chinese Medicine, Chengdu, China
}

Ulcerative colitis (UC) is a chronic inflammatory bowel disease impacting patients' quality of life and imposing heavy societal and economic burdens. Apoptosis of intestinal epithelial cells (IECs) has been considered an early event during the onset of UC and plays a crucial role in disease development. Thus, effectively inhibiting apoptosis of IECs is of critical significance for the clinical management of UC, presenting a potential direction for the research and development of pharmacotherapeutic agents. In recent years, research on the ameliorative effects of natural products on UC through inhibiting IECs apoptosis has attracted increasing attention and made remarkable achievements in ameliorating UC. In this review, we summarized the currently available research about the anti-apoptotic effects of natural products on UC and its mechanisms involving the death-receptor mediated pathway, mitochondrial-dependent pathway, ERS-mediated pathway, MAPK-mediated pathway, NF-kB mediated pathway, P13k/Akt pathway, JAK/STAT3 pathway, and NLRP3/ASC/Caspase-1 pathway. Hopefully, this review may yield useful information about the anti-apoptotic effects of natural products on UC and their potential molecular mechanisms and provide helpful insights for further investigations.

Keywords: ulcerative colitis, inflammatory bowel disease, apoptosis, natural products, intestinal epithelia cells

\section{INTRODUCTION}

Ulcerative colitis (UC) is a chronic inflammatory bowel disease characterized by relapsing and remitting mucosal inflammation restricted to the colon and rectum. It is a global health challenge, and its typical clinical presentation consists of diarrhea, hematochezia, and abdominal pain with histological features of diffuse inflammation limited to mucosa and submucosa, crypt abscesses, crypt architectural distortion, mucin depletion, and goblet cell depletion (Conrad et al., 2014; Yu and Rodriguez, 2017). The prevalence of UC varies in different regions. A systematic review of population-based studies demonstrated that the prevalence ranges from 1.2-57.3 per 100,000 in Asia to14.5-505.0 per 100,000 and 139.8-286.3 per 100,000 in Europe and North America, respectively ( $\mathrm{Ng}$ et al., 2017). UC can occur at any age and is most commonly diagnosed in the second to fourth decade of life (Du and Ha, 2020). Although the specific etiopathogenesis of UC remains obscure, it has been well-recognized that a complex interaction of intestinal microbiota, genetic susceptibility, and environmental factors may disturb the immune system and result in the immune-mediated chronic intestinal inflammatory response (Adams and Bornemann, 2013; Ananthakrishnan, 2015). The long-term inflammation leads to irreversible bowel damage and a higher risk of poor outcomes, such as colectomy and colorectal cancer (Rioux, 2008; Bopanna et al., 2017), impacting patients' quality of life, imposing heavy social and economic burdens, even 
increasing mortality. Conventional treatments for UC, including aminosalicylates, corticosteroids, and immunosuppressants, only induce and maintain remission and often elicit adverse effects (Wan et al., 2014; Burri et al., 2020). Several bioagents have been developed to target such molecular mechanisms as tumor necrosis factor (TNF), integrin, and Janus kinase (JAK) (Pugliese et al., 2017; Wehkamp and Stange, 2018). However, these therapeutic agents have primarily focused on the inflammatory cascades to alleviate the disease process rather than histological healing, and most of these substances are expensive for the medium- and low-income populations. Therefore, researching effective and affordable medications with fewer side effects for UA is urgently needed.

Previous studies have demonstrated that the apoptosis of intestinal epithelial cells (IECs) in the colon contributes to chronic inflammatory bowel diseases. IECs play a significant role in host defense, mucosal homeostasis maintenance, and immune response (Eissa et al., 2019; Zhang J. et al., 2020). The intestinal mucosa structure is maintained by a sensitive balance between the apoptosis and proliferation of epithelial cells, which may be disturbed in the inflammatory intestine due to the increased proinflammatory cytokines, including tumor necrosis factor (TNF), interleukin (IL), and interferon family members (Schulzke et al., 2006; Qiu et al., 2011). Apoptosis of IECs has been considered an early event during the onset of UC and plays a crucial role in disease development (Iwamoto et al., 1996). Both extrinsic and intrinsic apoptotic pathways are involved in the UC pathology and regulated by multiple signaling pathways. IECs act as a barrier between lumina and the external environment. Excessive epithelial apoptosis disrupts the epithelial defense system and may cause the breakdown of epithelial barrier function, which may facilitate the mucosal invasion of intraluminal microorganisms and luminal antigen uptake (Hagiwara et al., 2002; Schulzke et al., 2009; Seidelin and Nielsen, 2009; Araki et al., 2010) and potentiate the prolonged inflammatory response. Therefore, effectively inhibiting apoptosis of IECs and retaining the integrated epithelial barrier are of critical significance for the clinical management of UC, presenting a potential direction for the research and development of pharmacotherapeutic agents (Verstege et al., 2006).

Recently, natural products, including extracts and isolated metabolites from medicinal botanical drugs and plants, have drawn increasing attention for their potential therapeutic effects on UC with high availability and fewer side effects (Wan et al., 2014; Santana et al., 2017). These natural agents have been demonstrated to possess anti-inflammatory, antioxidative, anti-apoptotic, antiplatelet, and immune-regulatory properties through mediating multiple signaling pathways related to the pathogenesis of UC (Ke et al., 2012; Triantafyllidi et al., 2015; Cao et al., 2019). Furthermore, several extracts and isolated metabolites from natural products have been reported to exert amelioratory effects on UC through inhibiting IECs apoptosis with multiple pathways, including death receptor-mediated pathway, mitochondria-dependent pathway, endoplasmic reticulum stressmediated pathway, MAPK-mediated pathway, NF- $\mathrm{B}$ mediated pathway, and P13K/Akt mediated pathway. Therefore, to yield helpful insights for further research and development of novel and efficacious pharmaceutic intervention in this field, a comprehensive review of the anti-apoptotic activities of natural products on UC and their potential molecular mechanisms is necessary. In this review, the following electronic databases were searched from the inception to July 2021 to identify the eligible studies: PubMed, Embase, Web of Science, China National Knowledge Infrastructure, China Biomedical Literature Database, Wanfang Database, VIP database, and Chinese Scientific Journals Database. The following terms were used in a combination for the search: Colitis, Ulcerative, ulcerative colitis, inflammatory bowel disease, colitis, apoptosis, apoptotic, cell death, natural product, natural medicine, traditional medicine, and traditional Chinese medicine. A meticulous review was performed, and the quality of all the included studies was assessed in accordance with the Best practice in research-Overcoming common challenges in phytopharmacological research (Heinrich et al., 2020). The detailed information of natural products and their potential effects with mechanisms on modulating apoptosis in UC is illustrated in Tables 1, 2, and the chemical structures of isolated metabolites are summarized in Table 3.

\section{EFFECTS AND MECHANISMS OF NATURAL PRODUCTS ON APOPTOSIS IN UC}

\subsection{Death Receptor-Mediated Pathway}

The apoptosis pathway activated by death receptors is also known as the "extrinsic pathway". The ligand-bound death receptors refer to the proteins of tumor necrosis factor superfamily, such as tumor necrosis factor receptor, Fas, and tumor necrosis factorrelated apoptosis initiating ligand-receptor (Vanamee and Faustman, 2018). These receptors can be activated by binding to their ligands, such as TNF- $\alpha$, FasL, and TRAIL. The activated death receptors recruit Fas-associated death domain (FADD), the adaptor protein, which binds to the receptor and interacts with pro-caspase-8 to form a complex Death-inducing Signaling Complex (DISC), leading to the auto-cleavage and activation of caspase-8. Activated caspase- 8 initiates the executioner caspase- 3 to trigger the apoptotic cascades (Thorburn, 2004; Valmiki and Ramos, 2009). On the other hand, there is a cross-linking between the extrinsic pathway and intrinsic pathway (mitochondriadependent apoptotic pathway), in which caspase-8 plays a critical role. Bid, a member of the bcl- 2 family that regulates mitochondrial apoptosis, is cleaved by caspase- 8 to activate the subsequent apoptotic events (Kantari and Walczak, 2011). Previous studies have already found that death receptormediated apoptosis is involved in the mucosal defect in UC (Yan et al., 2001; Yukawa et al., 2002; Fan et al., 2020).

In 2011, Liu et al. reported that Sishen Pill (2.5-10 g/kg), a prescription from traditional Chinese medicine (TCM), could inhibit epithelial apoptosis in rats through down-regulating Fas/FasL and up-regulating bcl-2 in colon tissues (Liu et al., 2011). In the same year, a study by Liu et al. found that Iridoid Glycosides $(80-240 \mathrm{mg} / \mathrm{kg}$ ), a fraction of Folium syringae [Myrtaceae: Syringa vulgaris L.] leaves, ameliorated epithelial apoptosis in experimental colitis of rats by 
TABLE 1 | Anti-apoptotic activities of natural products on UC-induced intestinal epithelial apoptosis.

\begin{tabular}{|c|c|c|c|c|c|}
\hline $\begin{array}{l}\text { Potential } \\
\text { mechanisms }\end{array}$ & Detailed mechanisms & $\begin{array}{l}\text { Extracts/Isolated } \\
\text { metabolites } \\
\text { (dose/concentration) }\end{array}$ & Cells/Animal & $\begin{array}{l}\text { Related } \\
\text { targets }\end{array}$ & Refs \\
\hline \multirow[t]{4}{*}{$\begin{array}{l}\text { Death receptor } \\
\text { apoptotic pathway }\end{array}$} & $\begin{array}{l}\text { Down-regulating Fas and FasL; } \\
\text { Down-regulating caspase-3, -9, } \\
\text { and bax; Up-regulating bcl-2 }\end{array}$ & Baicalin (30-120 mg/kg) & $\begin{array}{l}\text { RAW264.7 cells stimulated by } \\
\text { lipopolysaccharide; } 2,4,6- \\
\text { trinitrobenzene sulfonic acid- } \\
\text { induced rats }\end{array}$ & $\begin{array}{l}\text { Fas, FasL, } \\
\text { caspase-3, -9, } \\
\text { bax, bcl-2 }\end{array}$ & Yao et al. (2016) \\
\hline & $\begin{array}{l}\text { Down-regulating Fas; Increasing } \\
\text { FasL, bcl-2 }\end{array}$ & Sishen Wan $(2.5,5,10 \mathrm{~g} / \mathrm{kg})$ & $\begin{array}{l}\text { Sprague-Dawley rats } \\
\text { stimulated by TNBS }\end{array}$ & Fas, FasL, bcl-2 & Liu et al. (2011) \\
\hline & $\begin{array}{l}\text { Down-regulating Fas, FasL, bax, } \\
\text { caspase-3; up-regulating bcl-2 }\end{array}$ & $\begin{array}{l}\text { Iridoid Glycosides (80, } 160 \text { and } \\
240 \mathrm{mg} / \mathrm{kg} \text { ) }\end{array}$ & $\begin{array}{l}\text { Sprague-Dawley rats } \\
\text { stimulated by DSS }\end{array}$ & $\begin{array}{l}\text { Fas, FasL, } \\
\text { caspase-3, } \\
\text { bcl-2 }\end{array}$ & Liu and Wang, (2011) \\
\hline & $\begin{array}{l}\text { Decreasing Fas, FasL, caspase- } \\
3 \text {, bax; Increasing bcl-2 }\end{array}$ & Wumei Pill (13.3-53.2 g/kg) & $\begin{array}{l}\text { Sprague-Dawley rats } \\
\text { stimulated by TNBS }\end{array}$ & $\begin{array}{l}\text { Fas, FasL, } \\
\text { caspase-3, bax, } \\
\text { bcl-2 }\end{array}$ & $\begin{array}{l}\text { (Shuguang et al., 2016; } \\
\text { Yi et al., 2016) }\end{array}$ \\
\hline
\end{tabular}

\begin{tabular}{|c|c|c|c|c|c|}
\hline $\begin{array}{l}\text { Mitochondria- } \\
\text { dependent }\end{array}$ & $\begin{array}{l}\text { Down-regulating bax; Up- } \\
\text { regulating bcl-2 }\end{array}$ & $\begin{array}{l}\text { Aucklandia and Coptis Pills } \\
(1.6 \mathrm{~g} / \mathrm{kg})\end{array}$ & Wistar rats stimulated by TNBS & $\mathrm{Bax}, \mathrm{bcl}-2$ & Yan and Jingen., (2016) \\
\hline \multirow[t]{10}{*}{ apoptotic pathway } & Down-regulating bax, caspase-3 & Hesperetin (100 mg/kg) & Wistar rats stimulated by TNBS & Bax, caspase-3 & $\begin{array}{l}\text { Polat and Karaboga, } \\
\text { (2019) }\end{array}$ \\
\hline & $\begin{array}{l}\text { Down-regulating cyt-c, caspase- } \\
9,-3, \text { bcl- } 2 / \text { bax }\end{array}$ & $\begin{array}{l}\text { Laggera Alata Flavone (100, } \\
200,400 \text { mg/kg) }\end{array}$ & $\begin{array}{l}\text { Sprague Dawley rats stimulated } \\
\text { by TNBS }\end{array}$ & $\begin{array}{l}\text { cyt-c, caspase- } \\
9,-3, \text { bcl-2, bax }\end{array}$ & $\begin{array}{l}\text { Xiaobin and Xiaodong., } \\
(2014)\end{array}$ \\
\hline & $\begin{array}{l}\text { Down-regulating bax; Up- } \\
\text { regulating bcl-2 }\end{array}$ & $\begin{array}{l}\text { Astragalus Polysaccharide } \\
(200 \mathrm{mg} / \mathrm{kg})\end{array}$ & Wistar rats stimulated by TNBS & Bax, bcl-2 & Weijie et al. (2019) \\
\hline & $\begin{array}{l}\text { Down-regulating bax, caspase- } \\
3 \text {; Up-regulating bcl-2 }\end{array}$ & Indigo (200, 400, 800 mg/kg) & $\begin{array}{l}\text { C57BL/ } 6 \text { mice stimulated } \\
\text { by DSS }\end{array}$ & $\begin{array}{l}\text { Bax, caspase-3, } \\
\text { bcl2 }\end{array}$ & Wenqiang et al. (2019) \\
\hline & $\begin{array}{l}\text { Decreasing bax mRNA } \\
\text { expression; Increasing bcl-2 } \\
\text { mRNA expression }\end{array}$ & Aloe Vera Gel (200 mg/kg) & $\begin{array}{l}\text { Sprague Dawley rats stimulated } \\
\text { by } 3 \% \text { acetic acid }\end{array}$ & Bax, bcl-2 & $\begin{array}{l}\text { Hassanshahi et al. } \\
\text { (2020) }\end{array}$ \\
\hline & Decreasing bax, caspase-3 & $\begin{array}{l}\text { Coptidis Rhizoma and } \\
\text { Magnoliae Officinalis Cortex ( } 1 \text {, } \\
2,4 \mathrm{~g} / \mathrm{kg})\end{array}$ & $\begin{array}{l}\text { Sprague Dawley rats stimulated } \\
\text { by TNBS }\end{array}$ & Bax, caspase-3 & Xian-juan et al. (2020) \\
\hline & $\begin{array}{l}\text { Up-regulating bcl-2; Down } \\
\text { regulating bax and caspase-3 }\end{array}$ & $\begin{array}{l}\text { Qingchang Wenzhong granule } \\
(0.42-2.20 \mathrm{~g} / \mathrm{kg})\end{array}$ & $\begin{array}{l}\text { Male Sprague Dawley rats } \\
\text { stimulated by DSS }\end{array}$ & $\begin{array}{l}\text { Bcl-2, bax, } \\
\text { caspase-3 }\end{array}$ & Shi et al. (2019) \\
\hline & $\begin{array}{l}\text { Down-regulating bax, caspase- } \\
\text { 3; Up-regulating bcl-2 }\end{array}$ & Artesunate (30 mg/kg) & $\begin{array}{l}\text { Female ICR mice stimulated } \\
\text { by DSS }\end{array}$ & $\begin{array}{l}\text { Bcl-2, bax, } \\
\text { caspase-3 }\end{array}$ & Yin et al. (2020) \\
\hline & $\begin{array}{l}\text { Decreasing bax, caspase-3; } \\
\text { Increasing bcl-2, bcl-xL }\end{array}$ & Plumericin $(0.5-2 \mu g ; 3$ mg/kg) & $\begin{array}{l}\text { IEC- } 6 \text { cells induced by LPS and } \\
\text { IFN; Male CD1 mice stimulated } \\
\text { by DNBS }\end{array}$ & $\begin{array}{l}\text { Bcl-2, bax, } \\
\text { caspase-3 }\end{array}$ & Rapa et al. (2021) \\
\hline & $\begin{array}{l}\text { Decreasing caspase-3, bax; } \\
\text { Increasing bcl,2 }\end{array}$ & Graviola (100 mg/kg) & $\begin{array}{l}\text { Male Wistar rats induced by } \\
\text { acetic acid }\end{array}$ & $\begin{array}{l}\text { caspase-3, bax, } \\
\text { bcl,2 }\end{array}$ & $\begin{array}{l}\text { Helal and Abd } \\
\text { Elhameed, (2021) }\end{array}$ \\
\hline \multirow{6}{*}{$\begin{array}{l}\text { Endoplasmic } \\
\text { reticulum stress- } \\
\text { mediated pathway }\end{array}$} & $\begin{array}{l}\text { Down-regulating GRP78, } \\
\text { caspase-3, -12 }\end{array}$ & $\begin{array}{l}\text { Berberine }(100,150 \\
200 \mathrm{mg} / \mathrm{kg} ; 10 \mathrm{ml} / \mathrm{kg})\end{array}$ & $\begin{array}{l}\text { Male BALB/c mice stimulated } \\
\text { by DSS }\end{array}$ & $\begin{array}{l}\text { GRP78, } \\
\text { caspase-3, -12 }\end{array}$ & $\begin{array}{l}\text { (Yan et al., 2018; Yan } \\
\text { et al., 2020) }\end{array}$ \\
\hline & $\begin{array}{l}\text { Decreasing GRP78, caspase- } \\
3,-12\end{array}$ & Glycyrrhizin $(0.5,1,2 \mathrm{mmol} / \mathrm{L})$ & $\begin{array}{l}\text { IECs induced by } \mathrm{H} 2 \mathrm{O} 2 ; \text { Male } \\
\text { BALB/c mice stimulated } \\
\text { by DSS }\end{array}$ & $\begin{array}{l}\text { GRP78, } \\
\text { caspase-3, -12 }\end{array}$ & Yan and Bin., (2020) \\
\hline & $\begin{array}{l}\text { Decreasing GRP78, PERK, } \\
\text { CHOP, caspase-3, - } 12\end{array}$ & $\begin{array}{l}\text { Ginsenoside Rb1 } \\
(20,40 \mathrm{mg} / \mathrm{kg})\end{array}$ & $\begin{array}{l}\text { C57BL/6 mice stimulated by } \\
\text { DSS; IEC- } 6 \text { rat intestinal } \\
\text { epithelial cells induced by TNBS }\end{array}$ & $\begin{array}{l}\text { GRP78, PERK, } \\
\text { CHOP, } \\
\text { caspase-3, }-12\end{array}$ & Dong et al. (2021) \\
\hline & $\begin{array}{l}\text { Inhibiting PERK-ATF4-CHOP } \\
\text { pathway }\end{array}$ & Limonin $(25,50,100$ mg/kg) & $\begin{array}{l}\text { Female C57BL/ } 6 \text { mice } \\
\text { stimulated by DSS; RAW } 264.7 \\
\text { cells induced by LPS }\end{array}$ & $\begin{array}{l}\text { p-PERK, } \\
p \text {-elF2 } \alpha, \text { ATF } 4 \\
\text { CHOP }\end{array}$ & Song et al. (2021) \\
\hline & $\begin{array}{l}\text { Decreasing p-PERK, } p \text {-elF2 } \alpha \text {, } \\
\text { ATF } 4, \text { CHOP, bax }\end{array}$ & $\begin{array}{l}\text { Gancao Xiexin Decoction (10, } \\
20,40 \mu \mathrm{L})\end{array}$ & $\begin{array}{l}\text { Caco- } 2 \text { cells Male BALB/c mice } \\
\text { stimulated by DSS }\end{array}$ & $\begin{array}{l}\text { PERK, elF2 } \alpha, \\
\text { ATF4, } \\
\text { CHOP, bax }\end{array}$ & Yan et al. (2021) \\
\hline & $\begin{array}{l}\text { Decreasing GRP78, CHOP, } \\
\text { PERK, elF2 } \alpha, \text { ATF4, XBP1s, } \\
\text { capsase-12 }\end{array}$ & Artesuante (30 mg/kg) & $\begin{array}{l}\text { Female ICR mice stimulated } \\
\text { by DSS }\end{array}$ & $\begin{array}{l}\text { GRP78, CHOP, } \\
\text { PERK, elF2 } \alpha \text {, } \\
\text { ATF4, XBP1s, } \\
\text { capsase-12 }\end{array}$ & Yin et al. (2021) \\
\hline \multirow[t]{2}{*}{$\begin{array}{l}\text { MAPK-mediated } \\
\text { pathway }\end{array}$} & $\begin{array}{l}\text { Suppressing p38, ERK } 1 / 2 \text {, and } \\
\text { MAP2K1 }\end{array}$ & $\operatorname{SNE}(50,200$ mg/kg) & $\begin{array}{l}\text { Male ICR mice stimulated } \\
\text { by DSS }\end{array}$ & $\begin{array}{l}\text { p38, ERK1/2, } \\
\text { MAP2K1 }\end{array}$ & Taya et al. (2016) \\
\hline & $\begin{array}{l}\text { Modulating p38-, JNK-MAPK } \\
\text { pathways }\end{array}$ & Curcumin (100 mg/kg) & $\begin{array}{l}\text { Male Wistar albino rats } \\
\text { stimulated by acetic acid }\end{array}$ & p38, JNK & $\begin{array}{l}\text { Topcu-Tarladacalisir } \\
\text { et al. (2013) } \\
\text { lued on following page) }\end{array}$ \\
\hline
\end{tabular}


TABLE 1 | (Continued) Anti-apoptotic activities of natural products on UC-induced intestinal epithelial apoptosis.

\begin{tabular}{|c|c|c|c|c|c|}
\hline $\begin{array}{l}\text { Potential } \\
\text { mechanisms }\end{array}$ & Detailed mechanisms & $\begin{array}{c}\text { Extracts/Isolated } \\
\text { metabolites } \\
\text { (dose/concentration) }\end{array}$ & Cells/Animal & $\begin{array}{l}\text { Related } \\
\text { targets }\end{array}$ & Refs \\
\hline & $\begin{array}{l}\text { Decreasing p38, p53, c-jun, } \\
\text { c-fos, bax, caspase-3; } \\
\text { Increasing bcl-2 }\end{array}$ & Si ShenWan (5 g/kg) & $\begin{array}{l}\text { C57/BL mice stimulated by } \\
\text { TNBS }\end{array}$ & $\begin{array}{l}\text { p38, c-jun, } \\
\text { c-fos, bax, } \\
\text { caspase-3, } \\
\text { bcl-2 }\end{array}$ & Zhao et al. (2013) \\
\hline & $\begin{array}{l}\text { Suppressing p38; down- } \\
\text { regulating caspase-3; up- } \\
\text { regulating PPAR } \gamma\end{array}$ & Geraniol (250 mg/kg) & $\begin{array}{l}\text { Male Wistar rats stimulated by } \\
\text { TNBS }\end{array}$ & $\begin{array}{l}\text { p38, caspase-3, } \\
\text { PPAR } \gamma\end{array}$ & Soubh et al. (2015) \\
\hline & $\begin{array}{l}\text { Inhibiting MAPK/NF-кB } \\
\text { pathway; Up-regulating bcl-2; } \\
\text { Down regulating bax and } \\
\text { caspase-3, -9 }\end{array}$ & $\begin{array}{l}\text { Paeoniflorin (15, 30, } 45 \text { mg/kg; } \\
2.5 \mathrm{~g} / \mathrm{kg})\end{array}$ & $\begin{array}{l}\text { Male Balb/c mice stimulated by } \\
\text { TNBS; Male Wistar rats } \\
\text { stimulated by DSS }\end{array}$ & $\begin{array}{l}\text { ERK, p38, blc-2, } \\
\text { bax, caspase- } \\
3,-9\end{array}$ & $\begin{array}{l}\text { (Gu et al., 2017; } \\
\text { (Lanzhen et al., 2020) }\end{array}$ \\
\hline & $\begin{array}{l}\text { Inhibiting MAPK/NF-кB } \\
\text { pathway; Increasing ERK1/2, } \\
p \text {-ERK, p38, p-p38, JNK, } \\
p \text {-JNK, } p \text {-IкB, p-p65 Decreasing } \\
\text { cleaved caspase-3; Increasing } \\
\text { bcl-2 }\end{array}$ & $\begin{array}{l}\text { Indirubin (10 mg/kg); Isatin } \\
(10 \mathrm{mg} / \mathrm{kg})\end{array}$ & $\begin{array}{l}\text { Male BALB/c mice stimulated } \\
\text { by DSS }\end{array}$ & $\begin{array}{l}\text { ERK, p38, JNK, } \\
\text { caspase-3, } \\
\text { bcl-2 }\end{array}$ & Gao et al. (2018) \\
\hline & $\begin{array}{l}\text { Increasing ERK1/2, p-ERK, p38, } \\
\text { p-p38, JNK, p-JNK, p-IкB, } \\
\text { p-p65 }\end{array}$ & $\begin{array}{l}\text { Chlorogenic Acid }(30,60 \text {, } \\
120 \mathrm{mg} / \mathrm{kg})\end{array}$ & $\begin{array}{l}\text { C57BL/6 mice stimulated } \\
\text { by DSS }\end{array}$ & $\begin{array}{l}\text { ERK, p38, } \\
\text { JNK, p65 }\end{array}$ & Gao et al. (2019) \\
\hline & $\begin{array}{l}\text { Inhibiting p-JNK, p-p38; } \\
\text { Increasing bcl-2; Decreasing bax }\end{array}$ & $\begin{array}{l}\text { Berberis lycium fruit extract } \\
(125-500 \mathrm{mg} / \mathrm{kg})\end{array}$ & Balb/C mice stimulated by DSS & $\begin{array}{l}\text { JNK, p38, bcl- } \\
2, \text { bax }\end{array}$ & Sharma et al. (2020) \\
\hline & $\begin{array}{l}\text { Inhibiting S100A9/MAPK/NF-кB } \\
\text { pathway; Increasing bcl-2; } \\
\text { Decreasing bax, caspase-3, p53 }\end{array}$ & Anemoside B4 $(5,10$ mg/kg) & SD rats stimulated by TNBS & $\begin{array}{l}\text { S100A9, TLR4, } \\
\text { JNK, p65, blc-2, } \\
\text { bax, caspase- } \\
\text { 3, p53 }\end{array}$ & $\begin{array}{l}\text { (Yong et al., 2020; } \\
\text { Zhang et al., 2021) }\end{array}$ \\
\hline
\end{tabular}

\section{NF-kB mediated Inhibiting 1 kB $\alpha$ degradation,} pathway

caspase-3 activation
Inhibiting $1 \kappa B a$ degradation;

Down-regulating caspase-3, -9

Inhibiting IKB $\alpha$, and IKK $\beta$ Down-

regulating Fas/FasL, bax,

caspase-3; Up-regulating bcl-2

Down-regulating NF-kBp65, bax, caspase-3; Up-regulating bcl-2

Decreasing p-p65, caspase-3

Inhibiting $p$-|кBa, $\mathrm{p}-\mathrm{p} 65$;

Decreasing caspase-3, -9;

Increasing bcl-2

Suppressing NF-kB

phosphorylation; Decreasing

PARP

Down-regulating NF-kB, bax;

Up-regulating bcl-2

Suppressing NF-kBp65, pNF-

кB, ERK1/2, COX-2 Down-

regulating caspase- 3

Down-regulating TLR4, NF-kB, caspase-3; Suppressing

NLPR3, cleaved caspase-1,

ASC mRNA

Inhibiting NF-кBp65, IкBa Downregulating bax, caspase- 3 , cyto-

c; Up-regulating bcl-2

Decreasing NF-кBp65, $p$-|KK $/$

IKK $\beta, p-\mid \mathrm{KB} \alpha / \mathrm{KB} \alpha$; Decreasing

cyt-c, caspase-3, -9, bcl-2/bax
Deoxyschisandrin $(1-5 \mu \mathrm{g} / \mathrm{ml})$

Corilagin $(7.5,15,30 \mathrm{mg} / \mathrm{kg})$

Iridoid Glycosides Fraction (80, 160 and $240 \mathrm{mg} / \mathrm{kg}$ )

Portulaca Extract $(100$ mg/kg)

QingBai decoction

$(0.0195 \mathrm{ml} / \mathrm{g})$

Gallic acid $(20,40,60 \mathrm{mg} / \mathrm{kg}$, $\mathrm{mg} / \mathrm{ml})$

C. arietinum ethanol Extract (100, $200 \mathrm{mg} / \mathrm{kg}$ )

Oleuropein $(350 \mathrm{mg} / \mathrm{kg})$

6,7-Dihydroxy-2,4-

Dimethoxyphenanthrene

$(60,120,240 \mathrm{mg} / \mathrm{kg})$

Canna x generalis L.H. Bailey rhizome extract (100,

$200 \mathrm{mg} / \mathrm{kg}$ )

Coptisine $(100 \mathrm{mg} / \mathrm{kg})$

Baicalin (30, 60, $90 \mathrm{mg} / \mathrm{kg})$
HCT116 cells induced by H2O2

Male C57BL/6 mice stimulated by DSS

Sprague-Dawley rats

stimulated by DSS

Female mice stimulated by DSS

C57/bL mice stimulated by DSS

Balb/c mice stimulated by TNBS HIEC-6 cells induced by IL-1 $\beta$

Male ICR mice stimulated by DSS

Male laboratory albino rats stimulated by acetic acid Male BALB/c mice stimulated by DSS

Mice stimulated by DSS

Male BALB/c mice stimulated by DSS

RAW264.7 cells induced by LPS; Sprague Dawley rats stimulated by TNBS

\section{c-fos, bax}

p38, caspase-3, Soubh et al. (2015) bax, caspase- (Lanzhen et al., 2020)

ERK, p38, JNK, Gao et al. (2018) caspase-3,

IкBa, caspase-3 Gu et al. (2010)

IкBa, caspase- Xiao et al. (2013)

3, -9

IкBa, IKK $\beta$, Fas, (Liu and Wang, 2011;

FasL, Bax, Zhang et al., 2020a) caspase-3, bcl-2 NF-kBp65, bax, Kong et al. (2018) bcl-2, caspase-3 NF-kBp65, caspase-3 IкBa, NF-kBp65, Zhu et al. (2019a) caspase-3, -9 , bcl-2 NF-kBp65, Kim et al. (2020) PARP

NF-kB, bax, Motawea et al. (2020) bcl-2

NF-кBp65, pNF- Li et al. (2021) $\kappa \mathrm{B}, \mathrm{ERK} 1 / 2$, COX-2,

caspase-3

TLR4, NF-kB, NLPR3, ASC mRNA, caspase-3, -1

NF-кBp65, lкBa, Wang et al. (2021) ax, caspase-3, cyto-c, bcl-2 $\mathrm{NF}-\kappa \mathrm{Bp} 65$, $\mathrm{IKBa}, \mathrm{IKK} \beta$, cytc, caspase-3, -9 , bcl-2, bax

(Continued on following page) 
TABLE 1 | (Continued) Anti-apoptotic activities of natural products on UC-induced intestinal epithelial apoptosis.

\begin{tabular}{|c|c|c|c|c|c|}
\hline $\begin{array}{l}\text { Potential } \\
\text { mechanisms }\end{array}$ & Detailed mechanisms & $\begin{array}{l}\text { Extracts/Isolated } \\
\text { metabolites } \\
\text { (dose/concentration) }\end{array}$ & Cells/Animal & $\begin{array}{l}\text { Related } \\
\text { targets }\end{array}$ & Refs \\
\hline & $\begin{array}{l}\text { Inhibiting TLR4, NF-кB; } \\
\text { Decreasing bax, caspase-3; } \\
\text { Increasing bcl-2 }\end{array}$ & $\begin{array}{l}\text { Deoxyschizandrin }(20,40 \text {, } \\
80 \mathrm{mg} / \mathrm{kg})\end{array}$ & $\begin{array}{l}\text { Sprague Dawley rats stimulated } \\
\text { by DSS }\end{array}$ & $\begin{array}{l}\text { TLR4, NF-kB, } \\
\text { bcl-2, bax, } \\
\text { caspase-3 }\end{array}$ & Yu and Qian, (2021) \\
\hline & $\begin{array}{l}\text { Decreasing NF-кBp65, } \\
\text { caspase-3 }\end{array}$ & $\begin{array}{l}\text { Hyperoside }(25,50, \\
100 \mathrm{mg} / \mathrm{kg})\end{array}$ & Wistar rats stimulated by TNBS & $\begin{array}{l}\mathrm{NF}-\kappa \mathrm{Bp} 65 \\
\text { caspase-3 }\end{array}$ & Yu et al. (2021) \\
\hline \multirow[t]{4}{*}{ P13K/Akt pathway } & $\begin{array}{l}\text { Regulating P13K/Akt pathway; } \\
\text { Decreasing caspase-9, FasL }\end{array}$ & Baicalin (20, 50, 100 mg/kg) & $\begin{array}{l}\text { Male Sprague Dawley rats } \\
\text { stimulated by TNBS }\end{array}$ & $\begin{array}{l}\text { P13K, Akt, } \\
\text { caspase-9, FasL }\end{array}$ & Zhu et al. (2017) \\
\hline & Regulating PI3K/Akt activation & $\begin{array}{l}\text { Oxymatrine }(25,50 \text {, } \\
100 \mathrm{mg} / \mathrm{kg})\end{array}$ & $\begin{array}{l}\text { Male BALB/c mice stimulated } \\
\text { by DSS }\end{array}$ & PI3K, Akt & Chen et al. (2017) \\
\hline & $\begin{array}{l}\text { Promoting P13K, Akt activity } \\
\text { Down-regulating caspase-3, } \\
\text { bad; Up-regulating bcl-2, p53 }\end{array}$ & $\begin{array}{l}\text { Costus root granules } \\
(1,000 \mathrm{mg} / \mathrm{kg})\end{array}$ & $\begin{array}{l}\text { Male Sprague Dawley rats } \\
\text { stimulated by DSS }\end{array}$ & $\begin{array}{l}\text { PI3K, Akt, } \\
\text { caspase-3, bcl- } \\
2 \text {, bax, p53 }\end{array}$ & Wang et al. (2018) \\
\hline & $\begin{array}{l}\text { Regulating Akt; Decreasing } \\
\text { caspase-3, -9, PARP }\end{array}$ & Luteolin (50, 100 mg/kg) & $\begin{array}{l}\text { Male C57BL/6 mice stimulated } \\
\text { by DSS }\end{array}$ & $\begin{array}{l}\text { Akt, caspase-3, } \\
-9, \text { PARP }\end{array}$ & Vukelic et al. (2020) \\
\hline \multirow[t]{10}{*}{ Other } & Decreasing caspase-3, -8 & $\begin{array}{l}\text { polysaccharide of Portulaca } \\
\text { oleracea ( } 200 \mathrm{mg} / 0.33 \mathrm{ml})\end{array}$ & $\begin{array}{l}\text { Male Sprague Dawley rats } \\
\text { stimulated by TNBS }\end{array}$ & Caspase-3, -8 & Feng et al. (2010) \\
\hline & Decreasing caspase-3 & Honey (5 g/kg) & $\begin{array}{l}\text { Male albino Wistar rats } \\
\text { stimulated by DSS }\end{array}$ & Caspase-3 & $\begin{array}{l}\text { Nooh and Nour-Eldien, } \\
\text { (2016) }\end{array}$ \\
\hline & Inhibiting JAK2/STAT3 pathway & $\begin{array}{l}\text { Aloe polysaccharide } \\
(15 \mathrm{mg} / \mathrm{kg})\end{array}$ & $\begin{array}{l}\text { HT- } 29 \text { cell induced by LPS; } \\
\text { Male SD rats stimulated by } \\
\text { TNBS }\end{array}$ & $\begin{array}{l}\text { JAK2, p-JAK2, } \\
\text { STAT3, } \\
\text { p-STAT3 }\end{array}$ & Lin et al. (2017) \\
\hline & $\begin{array}{l}\text { Up-regulating Sonic hedgehog } \\
\text { signaling pathway; Decreasing } \\
\text { caspase-3, bax; Increasing bcl-2 }\end{array}$ & Polydatin $(15,30,45$ mg/kg) & $\begin{array}{l}\text { Male C57BL/ } 6 \text { mice stimulated } \\
\text { by DSS }\end{array}$ & $\begin{array}{l}\text { Shh, caspase-3, } \\
\text { bcl-2, bax }\end{array}$ & Lv et al. (2018) \\
\hline & $\begin{array}{l}\text { Down-regulating } p-J A K 2 \text {, } \\
\text { pSTAT3, caspase-3, -9; Up- } \\
\text { regulating bcl-2, bcl-xL }\end{array}$ & $\begin{array}{l}\text { Tripterygium glycosides } \\
(27 \mathrm{mg} / \mathrm{kg})\end{array}$ & $\begin{array}{l}\text { Male Sprague Dawley rats } \\
\text { stimulated by TNBS }\end{array}$ & $\begin{array}{l}\text { JAK2, STAT3, } \\
\text { caspase-3, -9, } \\
\text { bcl-2, bcl-xL }\end{array}$ & Nan et al. (2019) \\
\hline & $\begin{array}{l}\text { Down-regulating bax; Up- } \\
\text { regulating bcl-2 }\end{array}$ & Hydroxytyrosol (50 mg/kg) & $\begin{array}{l}\text { Male laboratory albino rats } \\
\text { stimulated by acetic acid }\end{array}$ & Bcl-2, bax & Elmaksoud et al. (2021) \\
\hline & $\begin{array}{l}\text { Inhibiting IL-10/JAK1/STAT3 } \\
\text { pathway }\end{array}$ & $\begin{array}{l}\text { Chushi Jianpi decoction } \\
(1 \mathrm{ml} / \mathrm{kg})\end{array}$ & $\begin{array}{l}\text { BALB/c mice stimulated } \\
\text { by DSS }\end{array}$ & $\begin{array}{l}\text { IL-10, JAK, } \\
\text { STAT3 }\end{array}$ & Chen et al. (2021) \\
\hline & $\begin{array}{l}\text { Decreasing bax, caspase-3, } \\
\text { TLR4, MyD88; Increasing bcl-2 }\end{array}$ & Crocin $(0.05,0.1 \mathrm{~g} / \mathrm{kg})$ & $\begin{array}{l}\text { Male Sprague Dawley rats } \\
\text { stimulated by DSS }\end{array}$ & $\begin{array}{l}\text { Bax, caspase-3, } \\
\text { TLR4, MyD88, } \\
\text { bcl-2 }\end{array}$ & Yang et al. (2020) \\
\hline & $\begin{array}{l}\text { Decreasing bax, caspase-3, -9; } \\
\text { Increasing VLDLR, bcl-2 }\end{array}$ & Tanshinol (15, 30 mg/kg) & $\begin{array}{l}\text { Male C57BL/6J mice stimulated } \\
\text { by DSS }\end{array}$ & $\begin{array}{l}\text { VLDLR, bax, bcl- } \\
\text { 2, caspase-3, -9 }\end{array}$ & Zhu et al. (2021) \\
\hline & $\begin{array}{l}\text { Decreasing NRLP3, ASC, } \\
\text { caspase-1 }\end{array}$ & Walnut oil $(2.5 \mathrm{ml} / \mathrm{kg})$ & $\begin{array}{l}\text { Kunming male mice stimulated } \\
\text { by DSS }\end{array}$ & $\begin{array}{l}\text { NRLP3, ASC, } \\
\text { caspase-1 }\end{array}$ & Miao et al. (2021) \\
\hline
\end{tabular}

TNBS, trinitrobenzene sulfonic acid; PPARy, peroxisome proliferator activated receptor; CHOP, C/EBP, homologous protein; GRP78, glucose-regulated protein 78; PERK, protein kinase R-like ER, kinase; ATF4, activating transcription factor; JNK, c-jun N-terminal kinase; DSS, dextran sulfate sodium; DNBS, dinitrobenzenesulfonate; SNE, spirogyra neglecta extract; TLR4, toll-like receptor 4; IL-8, interleukin-8; PPAR- $\gamma$, peroxisome proliferator-activated receptor- $\gamma$; Cyto-c, cytochrome-c; LPS, lipopolysaccharide; TRAF6, tumor necrosis factor receptorassociated factor 6; NLRP3, NOD-like receptor protein; ASC, apoptosis-associated speck-like protein containing CARD; RJ, rumex japonicus houtt; JAK, janus kinase; STAT3, signal transducer and activator of transcription 3; IFN, interferon- $\gamma$; VLDLR, very low density lipoprotein receptor; Hh, Hedgehog.

modulating the expressions of Fas, FasL, caspase-3, bax, and bcl-2 (Liu and Wang, 2011). In 2016, a study by Yan et al. showed that a TCM prescription, Wumei Pill (13.3-53.2 g/kg), could inhibit the excessive apoptosis in colonic epithelial cells of rats via decreasing Fas, FasL, and caspase-3 (Shuguang. et al., 2016). Another study by Hui et al. also confirmed that Wumei Pill could decrease the bax expression and increase bcl2 expression, exerting anti-apoptotic effects on colonic epithelial cells (Yi. et al., 2016). In vitro and in vivo studies on baicalin, a bioactive constituent from the root of Scutellariae radix [Lamiaceae: Scutellaria baicalensis Georgi], showed its anti-apoptotic activity, and the potential mechanisms were correlated to the down-regulation of Fas and FasL (Yao et al., 2016). The effects and mechanisms of natural products on death receptors-mediated apoptosis of UC are summarized in Figure 1.

\subsection{Mitochondria-dependent Apoptotic Pathway}

Diverse apoptotic stimuli may evoke the decrease of mitochondrial membrane potential (MMP) and the release of pro-apoptotic proteins to activate the mitochondria-dependent apoptotic pathway (intrinsic pathway) (Thorburn, 2004; Elmore, 2007). The MMP integrity is regulated by the bcl-2 family composed of the proapoptotic members (e.g. bax, bad, bak, bim, and bid) and anti- 
TABLE 2 | Components of TCM prescriptions.

\begin{tabular}{|c|c|c|c|}
\hline Prescription & $\begin{array}{c}\text { Components [dosage(g)/ } \\
\text { Concentrations }(\%)]\end{array}$ & Scientific name & Refs \\
\hline Sishen Wan & $\begin{array}{l}\text { Wu Zhu Yu (6.67\%) } \\
\text { Bu Gu Zhi (26.67\%) } \\
\text { Wu Wei Zi (13.33\%) } \\
\text { Rou Dou Kou (13.33\%) } \\
\text { Sheng Jiang (26.67\%) } \\
\text { Da Zao (13.33\%) }\end{array}$ & $\begin{array}{l}\text { Rutaceae: Tetradium ruticarpum (A.Juss.) T.G. Hartley } \\
\text { Fabaceae: Cullen corylifolium (L.) Medik } \\
\text { Schisandraceae: Schisandra chinensis (Turcz.) Baill } \\
\text { Myristicaceae: Myristica fragrans Houtt } \\
\text { Zingiberaceae: Zingiber officinale Roscoe } \\
\text { Rhamnaceae: Ziziphus Jujuba Mill }\end{array}$ & $\begin{array}{l}\text { Liu et al. (2011), Zhao et al. } \\
\text { (2013) }\end{array}$ \\
\hline Wumei Pill & $\begin{array}{l}\text { Wu Mei }(16 \mathrm{~g}) \\
\text { Xi Xin }(6 \mathrm{~g}) \\
\text { Gan Jiang }(10 \mathrm{~g}) \\
\text { Gui Zhi }(6 \mathrm{~g}) \\
\text { Fu Zi }(6 \mathrm{~g}) \\
\text { Shu Jiao }(4 \mathrm{~g}) \\
\text { Huang Lian (16 g) } \\
\text { Ren Shen (6 g) } \\
\text { Dang Gui }(4 \mathrm{~g}) \\
\text { Huang Bo }(6 \mathrm{~g})\end{array}$ & $\begin{array}{l}\text { Rosaceae: Prunus mume (Siebold) Siebold and Zucc } \\
\text { Aristolochiaceae: Asarum heterotropoides F.Schmidt } \\
\text { Zingiberaceae: Zingiber officinale Roscoe } \\
\text { Lauraceae: Neolitsea cassia (L.) Kosterm } \\
\text { Ranunculaceae: Aconitum carmichaeli Debeaux } \\
\text { Rutaceae: Zanthoxylum bungeanum Maxim } \\
\text { Ranunculaceae: Coptis chinensis Franch } \\
\text { Araliaceae: Panax ginseng C.A.Mey } \\
\text { Apiaceae: Angelica sinensis (Oliv.) Diels } \\
\text { Rutaceae: Phellodendron amurense Rupr }\end{array}$ & Yi et al. (2016) \\
\hline $\begin{array}{l}\text { Qingchang Wenzhong } \\
\text { granule }\end{array}$ & $\begin{array}{l}\text { Huang Lian }(6 \mathrm{~g}) \\
\text { Pao Jiang }(10 \mathrm{~g}) \\
\text { Ku Shen }(15 \mathrm{~g}) \\
\text { Qing Dai }(6 \mathrm{~g}) \\
\text { Di Yu (15 g) } \\
\text { Mu Xiang (6 g) } \\
\text { San Qi }(6 \mathrm{~g}) \\
\text { Gan Cao }(6 \mathrm{~g})\end{array}$ & $\begin{array}{l}\text { Ranunculaceae: Coptis chinensis Franch } \\
\text { Zingiberaceae: Zingiber officinale Roscoe } \\
\text { Fabaceae: Sophora flavescens Aiton } \\
\text { Brassicaceae: Isatis tinctoria subsp. tinctoria } \\
\text { Rosaceae: Sanguisorba officinalis L } \\
\text { Asteraceae: Dolomiaea costus (Falc.) Kasana and A.K.Pandey } \\
\text { Araliaceae: Panax notoginseng (Burkill) F.H.Chen } \\
\text { Fabaceae: Glycyrrhiza glabra L }\end{array}$ & Shi et al. (2019) \\
\hline Gancao Xiexin decoction & $\begin{array}{l}\text { Gan Cao }(12 \mathrm{~g}) \\
\text { Gan Jiang }(9 \mathrm{~g}) \\
\text { Ban Xia }(9 \mathrm{~g}) \\
\text { Huang Qin }(9 \mathrm{~g}) \\
\text { Huang Lian }(3 \mathrm{~g}) \\
\text { Dang Shen }(9 \mathrm{~g}) \\
\text { Da Zao }(6 \mathrm{~g})\end{array}$ & $\begin{array}{l}\text { Fabaceae: Glycyrrhiza glabra L } \\
\text { Zingiberaceae: Zingiber officinale Roscoe } \\
\text { Araceae: Pinellia ternata (Thunb.) Makino } \\
\text { Lamiaceae: Scutellaria baicalensis Georgi } \\
\text { Ranunculaceae: Coptis chinensis Franch } \\
\text { Lamiaceae: Salvia miltiorrhiza Bunge } \\
\text { Rhamnaceae: Ziziphus Jujuba Mill }\end{array}$ & Yan et al. (2021) \\
\hline Qingbai decoction & $\begin{array}{l}\text { Da Qing Ye }(12 \mathrm{~g}) \\
\text { Ban Lan Gen }(20 \mathrm{~g}) \\
\text { Huang Bo }(9 \mathrm{~g}) \\
\text { Ku Shen }(20 \mathrm{~g}) \\
\text { Yi Ren }(30 \mathrm{~g}) \\
\text { Wu Zei Gu }(25 \mathrm{~g})\end{array}$ & $\begin{array}{l}\text { Brassicaceae: Isatis tinctoria subsp. Tinctoria, leaves } \\
\text { Brassicaceae: Isatis tinctoria subsp. Tinctoria, roots } \\
\text { Rutaceae: Phellodendron amurense Rupr } \\
\text { Fabaceae: Sophora flavescens Aiton } \\
\text { Poaceae: Coix lacryma-jobi L } \\
\text { Cuttlebone }\end{array}$ & Lin et al. (2019) \\
\hline Chushi Jianpi decoction & $\begin{array}{l}\text { Bai Zhu (5 g) } \\
\text { Cang Zhu (3 g) } \\
\text { Fu Ling (3 g) } \\
\text { Bai Shao (3 g) } \\
\text { Dang Gui (2 g) } \\
\text { Hou Po (2 g) } \\
\text { Chen Pi (2 g) } \\
\text { Zhu Ling (1.5 g) } \\
\text { Ze Xie }(1.5 \mathrm{~g}) \\
\text { Chai Hu (2 g) } \\
\text { Sheng Ma (2 g) } \\
\text { Fang Feng (2 g) } \\
\text { Gan Cao (1 g) }\end{array}$ & $\begin{array}{l}\text { Asteraceae: Atractylodes macrocephala Koidz } \\
\text { Asteraceae: Atractylodes lancea (Thunb.) DC. } \\
\text { Smilacaceae: Smilax glabra Roxb } \\
\text { Paeoniaceae: Paeonia lactiflora Pall } \\
\text { Apiaceae: Angelica sinensis (Oliv.) Diels } \\
\text { Magnoliaceae: Magnolia officinalis Rehder and E.H.Wilson } \\
\text { Rutaceae: Citrus x aurantium L } \\
\text { Pteridaceae: Adiantum capillus-veneris L } \\
\text { Alismataceae: Alisma plantago-aquatica L } \\
\text { Apiaceae: Bupleurum chinense DC. } \\
\text { Ranunculaceae: Actaea cimicifuga L } \\
\text { Apiaceae: Saposhnikovia divaricata (Turcz. ex Ledeb.) Schischk } \\
\text { Fabaceae: Glycyrrhiza glabra L }\end{array}$ & Chen et al. (2021) \\
\hline
\end{tabular}

apoptotic ones (e.g. bcl-2 and bcl-xL) (Jeong and Seol, 2008). Activating such apoptotic signaling pathways as p53-PUMA and death receptor pathways may enhance the pro-apoptotic proteins and reduce the anti-apoptotic proteins with a decrease in the MMP, disrupting the balance in the bcl-2 family. Increased mitochondrial membrane permeability induces the release of cytochrome-c (Cyt-c), which interacts with apoptosis protease-activating factor 1 (Apaf-1) to activate caspase-9 (Youle and Strasser, 2008; Qiu et al., 2011; Estaquier et al., 2012). Activated caspase- 9 initiates pro-caspase- 3 and -7 , and in turn, the activated caspase- 3 evokes pro-caspase- 9 , forming positive feedback. The activated executioner caspases cleave the downstream substrates, such as poly ADP-ribose polymerase (PARP), lamin, and fodrin, resulting in DNA fragmentation and apoptotic body formation (Fan et al., 2005). Increasing studies 
TABLE $3 \mid$ Chemical structures of natural products.

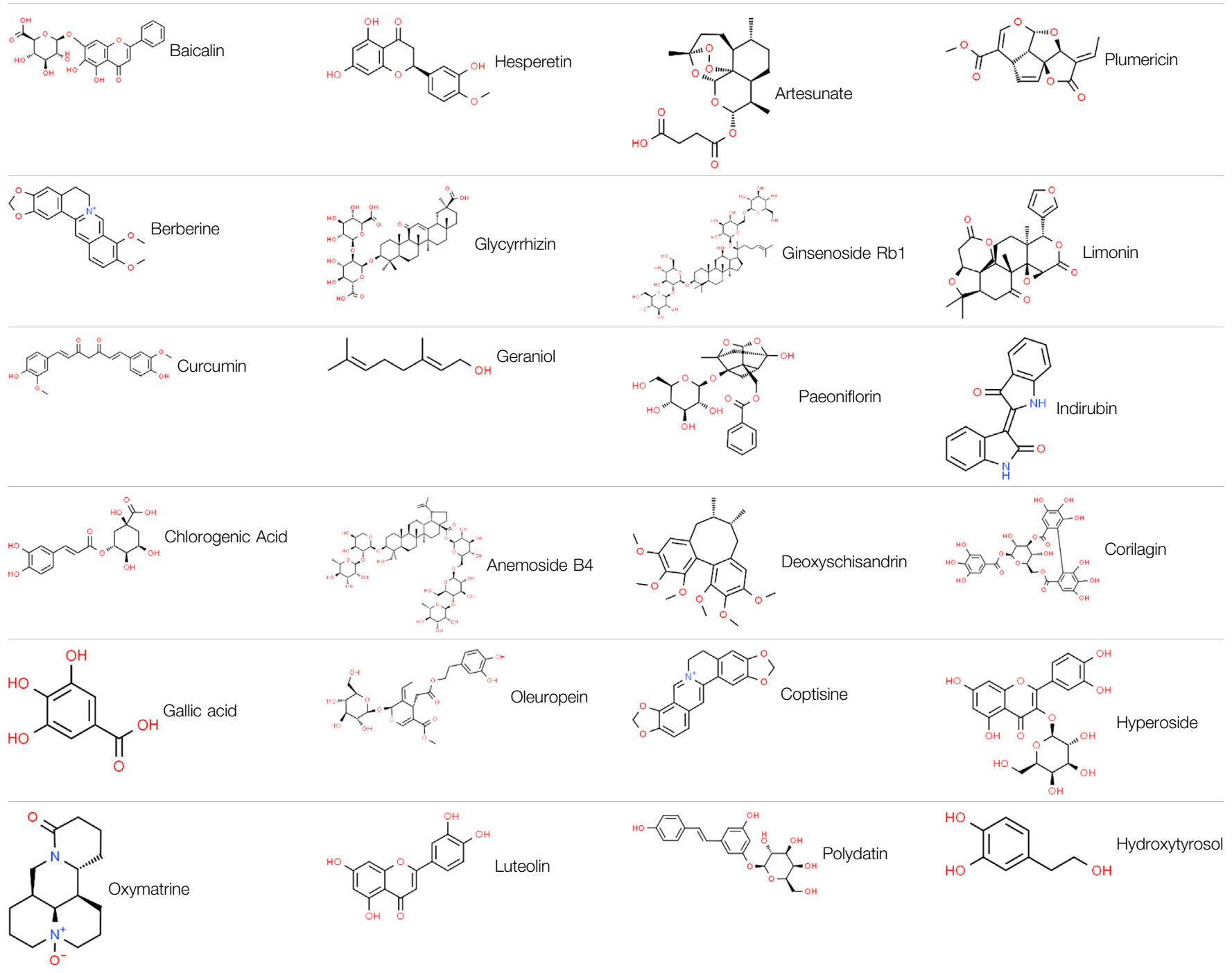

demonstrated that many natural products could mediate mitochondria-dependent apoptosis in UC. The effects and mechanisms of natural products on the mitochondria-dependent apoptotic pathway of UC are summarized in Figure 2.

\subsubsection{Extracts}

In 2014, a study by Zhao et al. revealed that Laggera Alata [Asteraceae: Laggera alata (D.Don) Sch. Bip. ex Oliv.] Flavone (LAF) $(100-400 \mathrm{mg} / \mathrm{kg})$ could dramatically reduce apoptosis of colonic epithelial cells in trinitro-benzene-sulfonic acid (TNBS)induced UC rats with the down-regulation of cyt-c, caspase-9, -3, and bcl-2/bax ratios (Xiaobin. and Xiaodong., 2014). In 2016, an investigation by Dong and Lu showed that Aucklandia [Asteraceae: Dolomiaea costus (Falc.) Kasana and A.K.Pandey] and Coptis [Ranunculaceae: Coptis chinensis Franch.] Pills (ACP) (1.6 g/kg) alleviated colonic epithelial apoptosis in TNBS-induced UC rats through decreasing bax expression and increasing bcl-2 expression (Yan. and Jingen., 2016). In 2018, Shi et al. found that a TCM clinical prescription, Qingchang Wenzhong granule (QCWZG), exerted anti-apoptotic effects $(0.42-2.20 \mathrm{~g} / \mathrm{kg})$ in attenuating DSS-induced colitis rats via inhibiting bax and caspase- 3 expressions and enhancing bcl-2 expression (Shi et al., 2019). In 2019, a study by $\mathrm{Ma}$ et al. indicated that Indigo [Brassicaceae: Isatis tinctoria subsp. tinctoria], one of the popular TCM botanical drugs, could reduce intestinal mucosa damage in experimental UC rats $(200-800 \mathrm{mg} / \mathrm{kg})$, and its mechanisms were associated with the down-regulation of caspase- 3 and bax and the up-regulation of bcl-2 (Wenqiang et al., 2019). In 2020, an experiment by Hassanshahi et al. demonstrated that Aloe Vera Gel (AVG) could reduce cell apoptosis in the colon of acetic-acid-reduced colitis rats with a decreased bax and increased bcl-2 expressions (Hassanshahi et al., 2020). Yang et al. reported that the combination treatment with Coptidis Rhizoma [Ranunculaceae: Coptis chinensis Franch.] and Magnoliae Officinalis Cortex [Magnoliaceae: Magnolia officinalis Rehder and E.H.Wilson] (1, 2, and $4 \mathrm{~g} / \mathrm{kg}$ ) could protect colonic mucosa from apoptosis by decreasing bax and caspase- 3 in TNBS- 


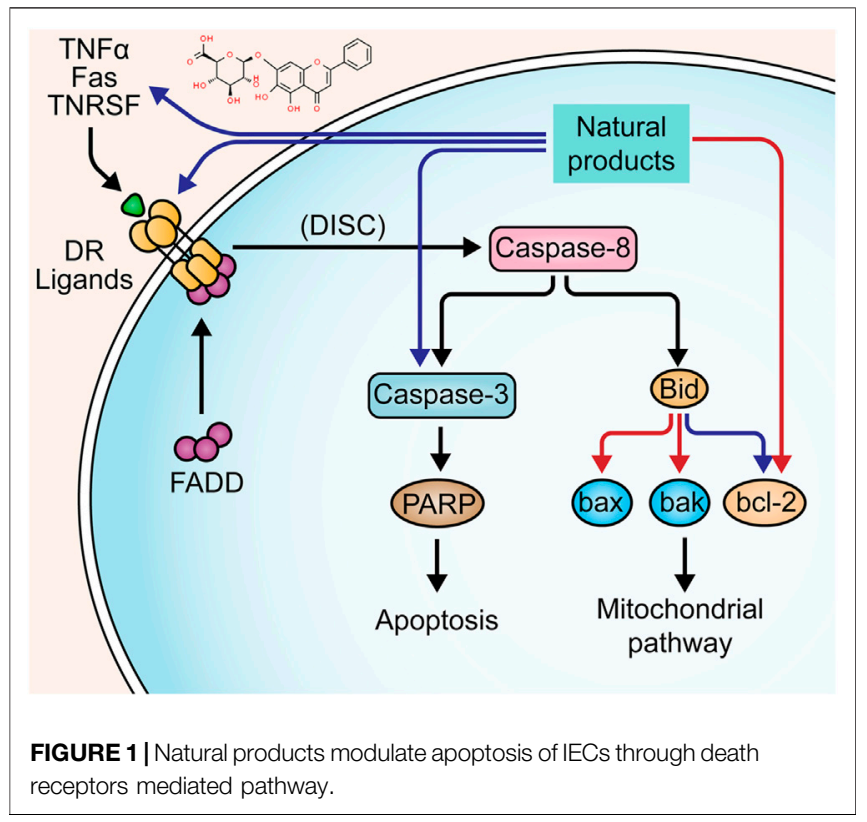

induced experimental rats (Xian-juan. et al., 2020). In 2021, Helal et al. elucidated the protective effects of graviola [Annonaceae: Annona muricata L.]. They observed that graviola treatment $(100 \mathrm{mg} / \mathrm{kg})$ attenuated apoptosis by modulating the expressions of bcl-2, bax, and caspase-3 (Helal and Abd Elhameed, 2021).

\subsubsection{Isolated Metabolites}

Hesperetin is a flavonoid compound found in many citrus fruits. In 2019, an investigation by Polat and Karaboga suggested that hesperetin treatment $(100 \mathrm{mg} / \mathrm{kg})$ could improve the histopathological changes in the colon mucosa of TNBS-induced UC rats through down-regulating bax and caspase-3 (Polat and Karaboga, 2019). In the same year, a study by Pan et al. showed that Astragalus Polysaccharide (AP) $(200 \mathrm{mg} / \mathrm{kg})$, one of the main constituents in Astragalus mongholicus [Fabaceae: Astragalus mongholicus Bunge], could alleviate colonic epithelial defect by decreasing bax expression and increasing bcl-2 expression (Weijie et al., 2019). Artesunate (ARS) is a semisynthetic derivative of Artemisinin. A study by Yin et al. demonstrated that ARS $(30 \mathrm{mg} / \mathrm{kg})$ suppressed apoptosis in colon tissues of DSS-induced colitis rats and notably protected epithelial integrity via inhibiting bax and caspase-3 and enhancing bcl-2 (Yin et al., 2020). In 2021, Plumericin, a major bioactive constituent of Himatanthus sucuuba [Apocynaceae: Himatanthus articulatus (Vahl) Woodson], was reported by Rapa to exert anti-apoptotic effects and protect the intestinal epithelium and its barrier function in vitro $(0.5-2 \mu \mathrm{g})$ and in vivo $(3 \mathrm{mg} / \mathrm{kg})$, and its potential mechanisms were correlated to the decrease of bax and caspase- 3 and the increase of bcl- 2 and bclxL (Rapa et al., 2021).

\subsection{Endoplasmic Reticulum Stress Mediated Pathway}

Disrupted epithelial cell populations and functions can affect mucosal homeostasis of UC, leading to Endoplasmic reticulum stress (ERS). The protein-folding capacity of the endoplasmic reticulum is decreased, causing the unfolded protein response (UPR). This process can up-regulate the expression of chaperone proteins-encoding genes, such as glucose- 2 regulated protein $78 \mathrm{kD}$ (GRP78) and Bip, triggering the downstream signaling of UPR,

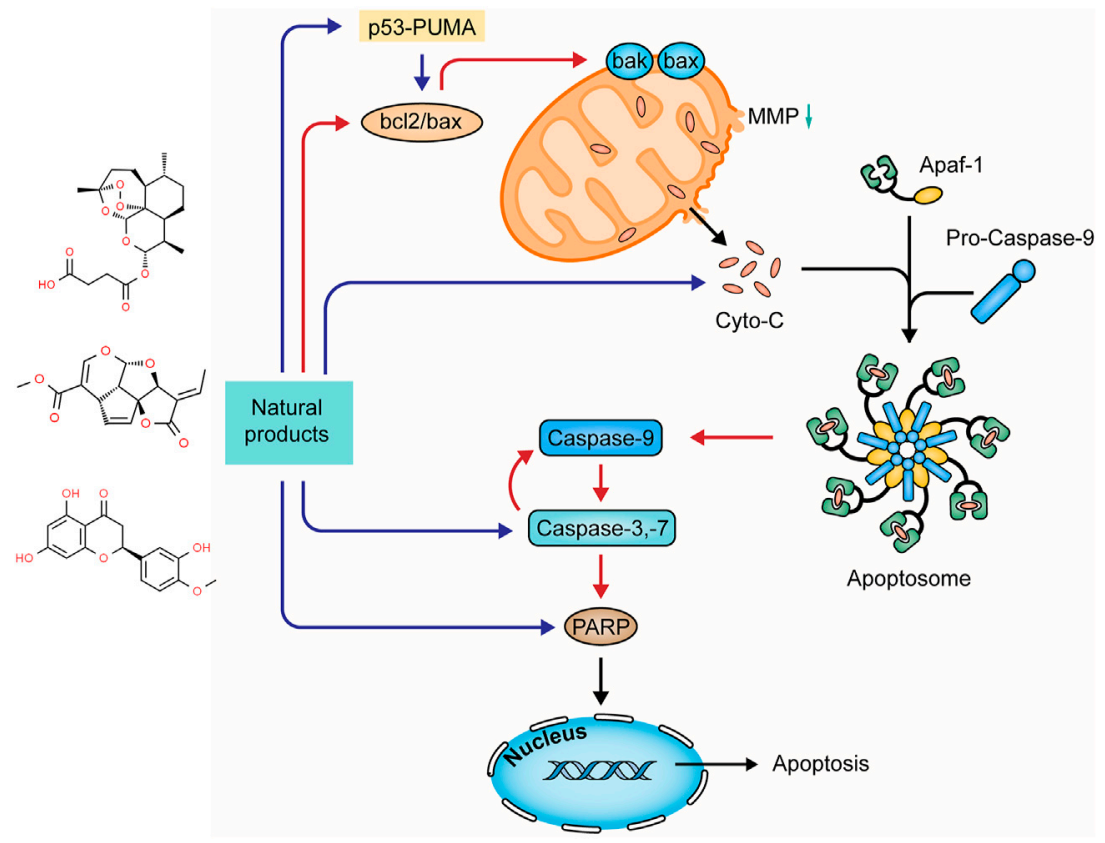

FIGURE 2 | Natural products modulate apoptosis of IECs through mitochondria-dependent pathway. 


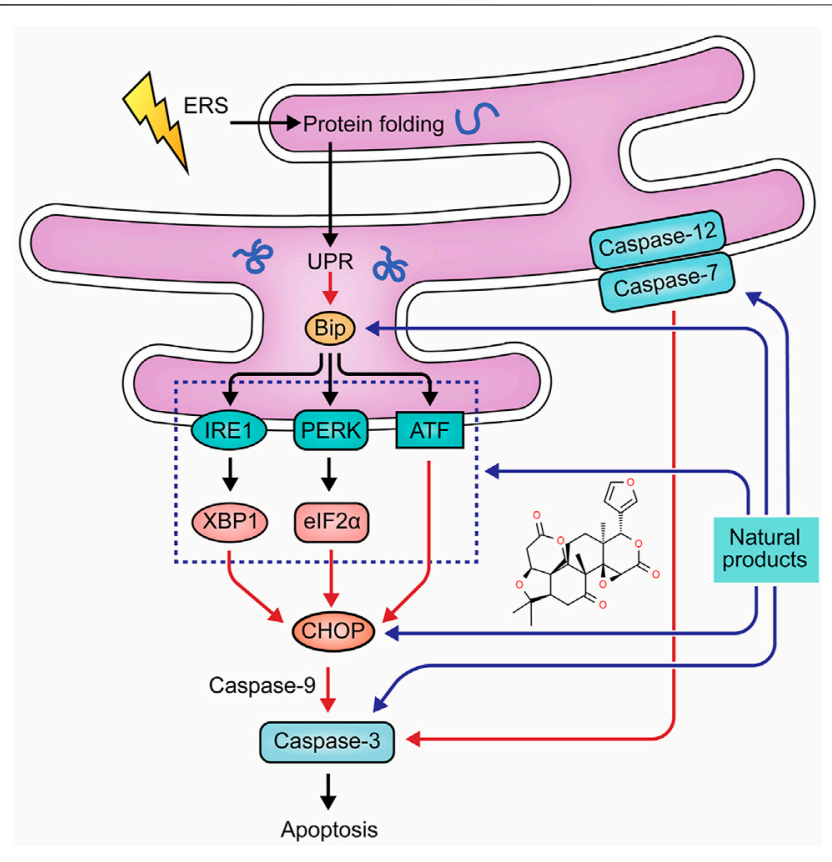

FIGURE 3 | Natural products modulate apoptosis of IECs through ERSmediated pathway.

namely IRE1-XBP1, PERK-eIF2 $\alpha$, and ATF pathway. Activation of these pathways contributes to an increase in the C/EBP-homologous protein (CHOP), the bcl-2-interacting mediator of cell death (Bim), and the p53 up-regulated modulator of apoptosis (PUMA) to promote apoptosis (Hetz, 2012; Cao, 2016). ERS also induces apoptosis through the caspase- 12 pathway. Pro-caspase- 12 is prelocated on the cytoplasmic side of the ER, which can be cleaved in response to ERS. Caspase-12 can activate caspase-3, -9, and -7, directly inducing apoptosis (Tan et al., 2006; Liu et al., 2013). The role of ERS in UC pathogenesis has been recognized for decades, and numerous studies have yielded considerable evidence that natural products can protect intestinal epithelial cells from UC-induced apoptosis. The improvement effects and potential mechanisms of natural products on ERS-mediated apoptosis are summarized in Figure 3.

\subsubsection{Extracts}

In 2021, Shen et al. found that Gancao Xiexin Decoction (10-40 $\mu \mathrm{L})$ could inhibit the activation of PERK- elF2 $\alpha-C H O P$ apoptotic signaling pathway to reduce the apoptosis of epithelial cells in UC, decreasing intestinal epithelial permeability and thus protecting intestinal mucosal barrier homeostasis (Yan et al., 2021).

\subsubsection{Isolated Metabolites}

Berberine (BBR) is one of the bioactive components in Coptis Chinensis [Ranunculaceae: Coptis chinensis Franch.]. In 2018, Shen et al. reported that BBR treatment $(100-200 \mathrm{mg} / \mathrm{kg})$ could decrease apoptosis in intestinal epithelial cells of UC rats, indicating that the anti-apoptotic effect of BBR was associated with the down-regulation of caspase-12 and -3 (Yan. et al., 2018). In 2020, Yan et al. further explored the anti-apoptotic

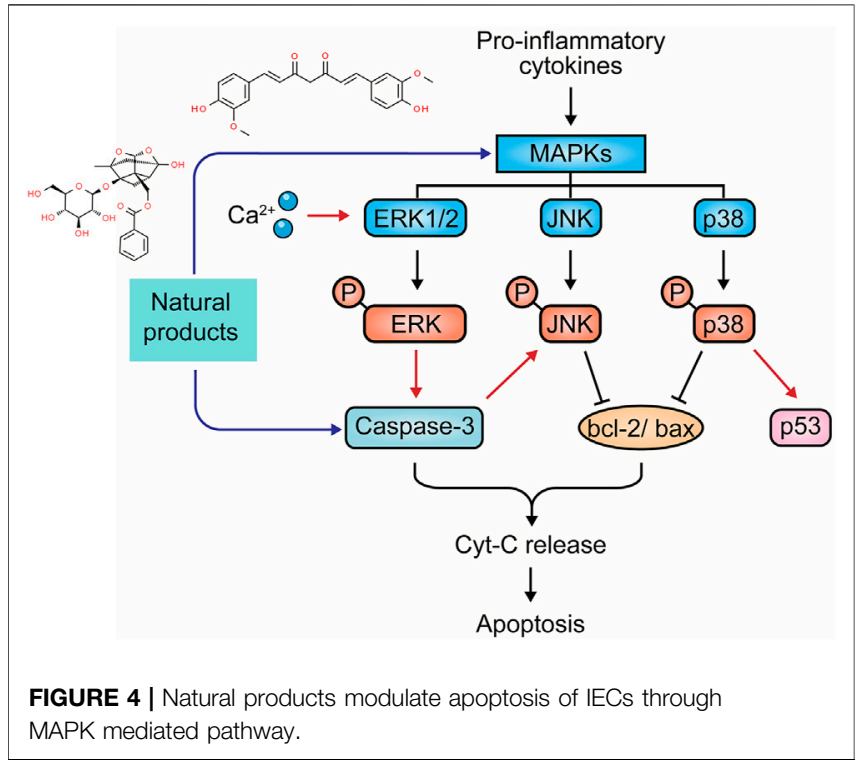

mechanisms of $\mathrm{BBR}$, and their results suggested that this component $(10 \mathrm{ml} / \mathrm{kg})$ also down-regulated GRP78 to alleviate UPR (Yan et al., 2020). In vitro and in vivo investigation of Shen et al. demonstrated glycyrrhizin $(0.5-2.0 \mathrm{mmol} / \mathrm{L})$ could regulate ERS-evoked intestinal epithelial apoptosis and protect cells from apoptosis by decreasing GRP78, caspase-12, and caspase-3 (Yan and Bin., 2020). In 2021, a study reported Ginsenoside Rb1 (20 and $40 \mathrm{mg} / \mathrm{kg}$ ), the major ginsenoside in ginseng [Araliaceae: Panax ginseng C.A.Mey.] with multiple pharmacological activities, significantly alleviated ERS in DSS-induced UC rats and TNBS-stimulated rat intestinal epithelial cells through decreasing GRP78, PERK, CHOP, caspase-12, and caspase-3 (Dong et al., 2021). Song et al. found that limonin, a tetracyclic triterpenoid compound obtained from plants of Rutaceae and Meliaceae, could improve colon pathology both in vitro and in vivo by inhibiting the PERK-ATF4-CHOP pathway to relieve ERS and subsequent cell apoptosis (Song et al., 2021). ARS has been demonstrated to alleviate UC by multiple pathways. Besides regulating mitochondria-dependent apoptosis, it also suppressed the activation of PERK-eIF2a-ATF4CHOP and IRE1a-XBP1 signaling pathways to prevent ERSmediated apoptosis in colon tissues (Yin et al., 2021).

\subsection{MAPK-Mediated Apoptotic Pathway}

Mitogen-activated protein kinase (MAPK) family members conventionally include extracellular-regulated kinase (ERK1/2), c-Jun N-terminal kinase (JNK), p38 MAPK, and ERK5 (Sun et al., 2015). They play pivotal roles in transduction extracellular stimuli into cellular responses of cell growth, migration, proliferation, differentiation, and apoptosis. ERK can be stimulated by growth factors and cytokines in UC pathology, leading to phosphorylation. The ERK signaling can play antiapoptotic and pro-apoptotic roles depending on the stimuli ( $\mathrm{Li}$ et al., 2014). JNK and p38 MAPK regulate several bcl-2 family proteins. One of the best-known transcription factors, p53, is also modulated by JNK/p38MAPK cascades to promote apoptosis. In 


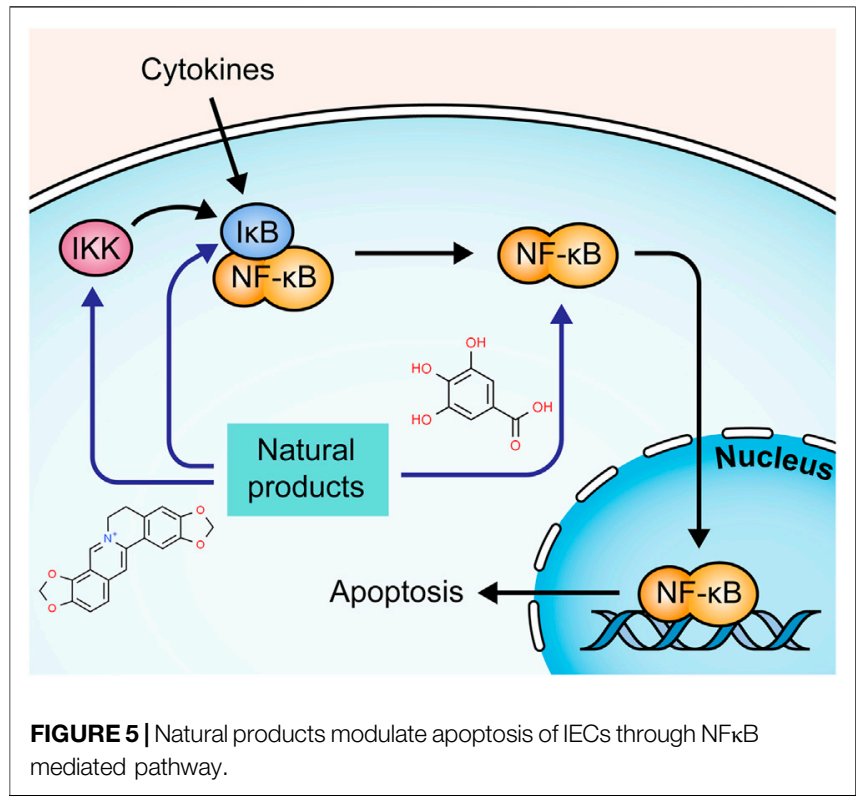

addition, JNK/p38MAPK has been reported to be associated with the activation of caspase cascades (Yue and Lopez, 2020). Currently, MAPKs are considered a potential target in the treatment of UC. The potential effectiveness and mechanism of natural products on MAPK-mediated apoptosis are summarized in Figure 4.

\subsubsection{Extracts}

Sishen Wan (SSW) is a notable TCM formula and has often been used in UC treatment. In 2013, Zhao et al. reported that SSW intervention $(5 \mathrm{~g} / \mathrm{kg})$ in TNBS-induced colitis rats decreased colonic epithelial apoptosis by suppressing p38MAPK, p53, caspase-3, c-jun, c-fos, and bax expressions (Zhao et al., 2013). In 2016, a study by Taya et al. demonstrated that the extract (50 and $200 \mathrm{mg} / \mathrm{kg}$ ) of Spirogyra neglecta, a freshwater green alga in the northern provinces of Thailand, diminished apoptosis of colonic epithelial cells in colitis mice via suppressing p38, ERK1/2, and MAP2K1 (Taya et al., 2016). Indirubin and Isatin are bioactive components in Qin Dai [Brassicaceae: Isatis tinctoria subsp. tinctoria]. In 2018, an investigation by Gao et al. demonstrated that the combination of Indirubin and Isatin inhibited cell apoptosis in DSS-induced UC mice through mediating the MAPK pathway, decreasing caspase-3, and increasing bcl-2 (Gao et al., 2018). Qing Chang Hua Shi granule (QCHS) also showed inhibitory effects on reducing UCinduced colonic apoptosis in vitro and in vivo by mediating MEK/ ERK pathway and decreasing the expression of such apoptosisrelated proteins as bax, bcl-2, caspase-3, -9, Fas, and Fas-L (Zhu et al., 2019a). In 2020, Sharma et al. reported that the extract of Berberis lycium Royle [Berberidaceae] fruit $(125-500 \mathrm{mg} / \mathrm{kg})$ could modulate intestinal epithelial cell apoptosis in mice through the inhibition of $p$-JNK and p-p38, increase of bcl-2, and decrease of bax, suggesting that it might be a viable candidate for UC treatment (Sharma et al., 2020).

\subsubsection{Isolated Metabolites}

Curcumin is a major constituent of medicinal turmeric [Zingiberaceae: Curcuma longa L.]. A study in 2013 revealed that curcumin $(100 \mathrm{mg} / \mathrm{kg})$ could reduce colon injury in UC rats through the modulation of p38-and JNK-MAPK pathways (Topcu-Tarladacalisir et al., 2013). In 2015, Soubh et al. reported that Geraniol, a natural monoterpene alcohol $(250 \mathrm{mg} / \mathrm{kg})$, hindered apoptosis in TNBS-induced UC rats by suppressing p38 and caspase-3 expressions. Ger also up-regulated PPAR $\gamma$, a transcriptional factor whose down-regulation is highly associated with the activation of the p38MAPK pathway (Soubh et al., 2015). Paeoniflorin (PA) is one of the major bioactive components in Paeony [Paeoniaceae: Paeonia lactiflora Pall.] root. In 2017, $\mathrm{Gu}$ et al. revealed that PA treatment $(15-45 \mathrm{mg} / \mathrm{kg})$ for experimental colitis mice could ameliorate the apoptosis in colitis tissues through inhibiting MAPK/NF- $\kappa \mathrm{B}$ pathway (Gu et al., 2017). Moreover, a study by Li et al., in 2020 showed that PA down-regulated bax, caspase-3, and caspase- 9 and up-regulated bcl-2 to protect UC-induced apoptosis (Lanzhen. et al., 2020). Chlorogenic acid is found in coffee and various TCM botanical drugs, such as honeysuckle [Caprifoliaceae: Lonicera japonica Thunb.], hawthorn [Rosaceae: Crataegus pinnatifida Bunge], eucommia [Eucommiaceae: Eucommia ulmoides Oliv.], and chrysanthemum [Asteraceae: Chrysanthemum $x$ morifolium (Ramat.) Hemsl.]. Gao et al. reported that Chlorogenic acid $(30-120 \mathrm{mg} / \mathrm{kg})$ could significantly alleviate colonic tissue apoptosis and inflammation via the mediation of the MAPK/ ERK/JNK signaling pathway (Gao et al., 2019). Anemoside B4, a bioactive triterpenoid saponin isolated from Chinese pulsatilla [Ranunculaceae: Pulsatilla chinensis (Bunge) Regel], was demonstrated to exert anti-apoptotic effects on UC through inhibiting p53, caspase-3, and bax expressions and the S100A9/MAPK/NF-kB signaling pathway (Yong. et al., 2020; Zhang et al., 2021).

\subsection{NF-кB Mediated Apoptotic Pathway}

The transcription factor nuclear factor-kappaB $(\mathrm{NF}-\kappa \mathrm{B})$ is also involved in the regulation of cell death. Under resting conditions, $\mathrm{NF}-\kappa \mathrm{B}$ is sequestered in the cytoplasm through interaction with $\mathrm{I} \kappa \mathrm{B}$, an inhibitory protein. In the presence of NF- $\mathrm{BB}$-activating stimuli, such as proinflammatory cytokines, I $\mathrm{B}$ can be phosphorylated by IKB kinase (IKK) and degraded, leading to the translocation of NF- $\kappa \mathrm{B}$ to the nucleus (Heyninck and Beyaert, 2001; Aranha et al., 2007). Activated NF- $\kappa B$ contributes to the transcription of multiple genes to regulate apoptosis induced by extrinsic and intrinsic apoptotic pathways (Baldwin, 2012; Liu et al., 2012). The potential mechanisms of natural products on NF- $\kappa \mathrm{B}$ mediated apoptosis are summarized in Figure 5.

\subsubsection{Extracts}

A study by Liu and Wang showed that the Iridoid Glycosides fraction inhibited $\mathrm{IkB} a$ phosphorylation and IKK activity in intestinal epithelial cells, regulating the NF- $\mathrm{kB}$ signaling pathway (Liu and Wang, 2011). Another study by Zhang et al. presented similar results of IG in regulating the NF- $\kappa B$ signaling 


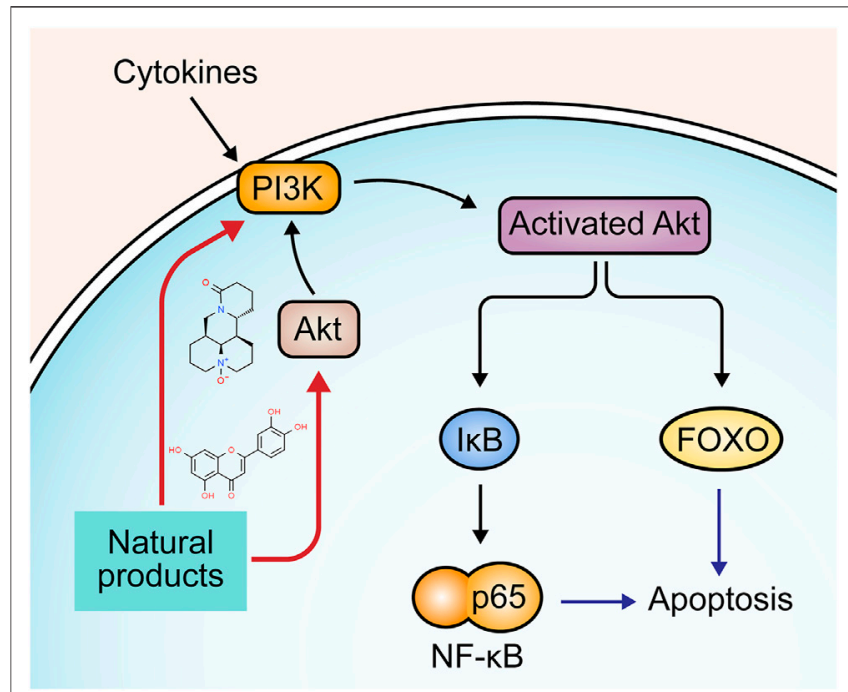

FIGURE 6 | Natural products modulate apoptosis of IECs through P13K/Akt pathway.

pathway (Zhang Y. et al., 2020). Corilagin is a major Gallotannin found in many medicinal plants. In 2013, a study by Xiao et al. demonstrated that Corilagin $(7.5-30 \mathrm{mg} / \mathrm{kg})$ suppressed the degradation of I $\mathrm{I} \mathrm{B} \alpha$ and down-regulated caspase-3 and -9 , reducing apoptosis in colon tissues of UC mice (Xiao et al., 2013). Portulaca [Portulacaceae: Portulaca oleracea L.] is a wildly used botanical drug in TCM. In 2018, an investigation by Kong et al. revealed that Portulaca extract could alleviate colitis in mice and mediate colonic cell apoptosis through inhibiting the NF- $\kappa \mathrm{B}$ pathway with decreased expressions of bax and caspase- 3 and increased expression of bcl-2 (Kong et al., 2018). A study by Lin et al. displayed that QingBai decoction (QBD), a TCM prescription, effectively reduced apoptosis in the colon of DSSinduced UC mice via regulating the NF- $\kappa B$ pathway and decreasing caspase-3 (Lin et al., 2019). Gallic acid is widely present in many plants and fruits. In 2019, Zhu et al. found that it inhibited UC-induced apoptosis in vitro and in vivo by suppressing the expressions of $p$-I $\kappa \mathrm{B} \alpha$ and $\mathrm{p}-\mathrm{NF}-\kappa \mathrm{B}$, decreasing caspase-3 and -9, and increasing bcl-2 (Zhu et al., 2019b). Chickpea [Fabaceae: Cicer arietinum L.] is a staple food crop in tropical and subtropical areas. In 2020, Kim et al. reported that its ethanol extract (100 and $200 \mathrm{mg} / \mathrm{kg}$ ) exerted a protective effect on DSS-induced apoptosis in colon tissue by the inhibition of nuclear factor-kappa B (NF- $\mathrm{B})$ and signal transducer and activator of transcription 3 (STAT3) (Kim et al., 2020). Canna [Cannaceae: Canna $x$ generalis L.H. Bailey] is wildly used in folk medicine for the treatment of many diseases. In 2021, a study by Mahmoud et al. showed that its rhizome ethanol extract (100, $200 \mathrm{mg} / \mathrm{kg}$ ) down-regulated NF- $\mathrm{\kappa B}$ and caspase- 3 expressions in colon tissues of colitis mice (Mahmoud et al., 2021).

\subsubsection{Isolated Metabolites}

In 2010, Gu et al. demonstrated that Deoxyschisandrin $(5 \mu \mathrm{g} / \mathrm{ml})$, one of the lignan components of Schisandra Chinensis [Schisandraceae: Schisandra chinensis (Turcz.) Baill.] fruits, could inhibit apoptosis of intestinal epithelial cells, and the potential mechanisms were associated with the inhibition of $\mathrm{I} \kappa \mathrm{B}$ degradation and the subsequent NF- $\kappa \mathrm{B}$ activation $(\mathrm{Gu}$ et al., 2010). A study by Shen et al. discovered that Baicalin (30-90 $\mathrm{mg} / \mathrm{kg}$ ) presented a significant anti-apoptotic effect on TNBS-induced UC rats and LPS-induced RAW264.7 cells through regulating the $\mathrm{IKK} / \mathrm{IKB} / \mathrm{NF}-\mathrm{kB}$ signaling and the expressions of such apoptosis-related proteins as cyt-c, caspase-3, -9, bcl-2, and bax (Shen et al., 2019). In 2020, Motawea et al. showed that Oleuropein $(350 \mathrm{mg} / \mathrm{mg})$, a major component of Olea europaea L.[Oleaceae], reduced apoptosis in colon tissues of experimental UC rats via down-regulating the expression of NF-kB and bax and up-regulating bcl-2 (Motawea et al., 2020). In 2021, a study by $\mathrm{Li}$ et al. revealed that 6,7Dihydroxy-2,4-Dimethoxyphenanthrene (CYP4, 60-240 mg/kg) from Chinese Yam [Dioscoreaceae: Dioscorea oppositifolia L.] could protect intestinal mucosa from apoptosis in DSS-induced colitis mice by suppressing NF- $\kappa \mathrm{B}$ and caspase-3 expressions ( $\mathrm{Li}$ et al., 2021). In the same year, Wang et al. stated that Coptisine $(100 \mathrm{mg} / \mathrm{kg})$, a major bioactive component from Rhizoma Coptidis [Ranunculaceae: Coptis chinensis Franch.], markedly alleviated DSS-induced apoptosis in intestinal epithelial cells of rats by restraining $\mathrm{I} \kappa \mathrm{B} \alpha$ phosphorylation and $\mathrm{NF}-\kappa \mathrm{B}$ translocation, down-regulating bax and caspase-3, and upregulating bcl-2 (Wang et al., 2021). Yu et al. observed that hyperoside $(25-100 \mathrm{mg} / \mathrm{kg})$, a flavonol glycoside isolated from plants of Hypericum and Crataegus, could inhibit TNBS-induced intestinal epithelial apoptosis in rats via decreasing NF- $\kappa B$ and caspase-3 (Yu et al., 2021). Yu and Qian reported that Deoxyschizandrin treatment $(20-80 \mathrm{mg} / \mathrm{kg})$ could reduce apoptosis of colonic epithelial cells in UC model mice, which might be attributed to the inhibition of TLR4/NF- $\kappa B$ signaling pathway and the regulation of bcl-2, bax, and caspase-3 (Yu and Qian, 2021).

\subsection{P13K/Akt Pathway}

Phosphatidylinositol 3-kinase (PI3K)/Akt signaling is implicated in multiple cellular processes, such as survival, proliferation, differentiation, and apoptosis (Vivanco and Sawyers, 2002). $\mathrm{P} 13 \mathrm{~K}$ can be activated by various cytokines and be recruited to the membrane. Akt, the downstream target protein of $\mathrm{P} 13 \mathrm{~K}$, migrates to the membrane and activates the sequential phosphorylation of $\mathrm{P} 13 \mathrm{~K}$. Activated Akt releases from the membrane to cytosol to phosphorylate fork-head transcription factor (FOXO), triggering the downstream signaling pathways that regulate many apoptotic genes related to the intrinsic and extrinsic pathway (Franke et al., 2003; Fresno Vara et al., 2004; Zhang et al., 2011). P13K/Akt can also activate NF- $\kappa B$ through phosphorylating IкB (Chen et al., 2017). The involvement of the P13K/Akt signaling pathway in UC pathogenesis has been welldocumented (Huang et al., 2011). Recently, compelling evidence has revealed that several natural products alleviate apoptosis in UC through regulating P13K/Akt signaling pathway. The potential mechanisms of natural products on P13K/Akt mediated apoptosis are summarized in Figure 6.

In 2017, Chen et al. reported that Oxymatrine $(25-100 \mathrm{mg} / \mathrm{kg})$, an alkaloid derived from the root of the 
Sophora flavescens [Fabaceae: Sophora flavescens Aiton], could alleviate apoptosis through P13K/Akt pathway and exhibit potential therapeutic effects against DSS-induced colitis (Chen et al., 2017). In the same year, another study by Zhu et al. found that baicalin (20-100 $\mathrm{mg} / \mathrm{kg}$ ) reduced the expression of caspase- 9 and FasL by regulating the P13K/Akt pathway (Zhu. et al., 2017). In 2018, an investigation by Wang et al. demonstrated that granules extracted from Costus root [Asteraceae: Dolomiaea costus (Falc.) Kasana and A.K.Pandey] (1000 mg/kg) could inhibit apoptosis of intestinal epithelial cells in UC rats via promoting the activities of $\mathrm{P} 13 \mathrm{~K}$ and Akt and subsequently down-regulating caspase- 3 and bad while up-regulating bcl-2 and p53 (Wang et al., 2018). Luteolin is a common flavonoid in several plants, such as lemon, apple, and onion. In 2020, Vukelic et al. showed that luteolin $(50-100 \mathrm{mg} / \mathrm{kg}$ ) decreased caspase- 3 , -9 , and PARP in UC mice, which may be related to Akt regulation (Vukelic et al., 2020).

\subsection{Other Reported Pathways}

Besides the major apoptotic pathways mentioned above, other mechanisms are also attributable to the anti-apoptotic activities of natural products on UC. For example, Aloe polysaccharide (AP) extracted from aloe vera [Asphodelaceae: Aloe vera (L.) Burm. f.] could effectively reduce the apoptosis in colonic tissues via inhibiting the JAK2/STAT-3 signaling pathway in vivo and vitro (Lin et al., 2017). Another study conducted in 2019 by $\mathrm{Bu}$ et al. revealed that Tripterygium glycosides $(27 \mathrm{mg} / \mathrm{kg})$ could attenuate intestinal mucosal apoptosis in UC rats through JAK2/ STAT3 signaling pathway (Nan et al., 2019). Moreover, an investigation by Chen et al. demonstrated that Chushi Jianpi Decoction, a TCM prescription, could reduce intestinal epithelial apoptosis in colitis mice through inhibiting IL-10/JAK1/STAT3 pathway (Chen et al., 2021). Polydatin (15-45 mg/kg), a main component in Polygonum cuspidatum [Polygonaceae: Reynoutria japonica Houtt.], was reported to exert protective effects on DSS-induced apoptosis in mice by up-regulating the Sonic hedgehog signaling pathway, decreasing caspase- 3 and bax, and increasing bcl-2 ( $\mathrm{Lv}$ et al., 2018). In 2020, Yang et al. demonstrated that crocin $(0.05-0.1 \mathrm{~g} / \mathrm{kg})$, a carotenoid compound derived from Crocus sativus L.(Iridaceae), could decrease bax and caspase 3 and increase bcl-2 in the intestinal tissue of UC rats, and its potential mechanisms might be correlated with the down-regulation of the TLR4/MyD88 signaling pathway (Yang et al., 2020). In 2021, a study by Miao et al. revealed that Walnut oil $(2.5 \mathrm{mg} / \mathrm{kg})$ downregulated the related gene proteins expression of the NLRP3/ ASC/Caspase-1 pathway to inhibit apoptosis in DSS-induced colitis mice (Miao et al., 2021).

In addition, some natural agents have exerted therapeutic effects on UC-elicited apoptosis, but their mechanisms need to be further verified. In 2010, Pan et al. reported that polysaccharides of Portulaca oleracea $(200 \mathrm{mg} / 0.33 \mathrm{ml})$ could reduce intestinal epithelial apoptosis in TNBS-induced UC rats with decreased caspase- 3 and -8 expression in the epithelium (Feng. et al., 2010). Interestingly, feeding DSS-induced colitis rats with honey $(5 \mathrm{~g} / \mathrm{kg})$ could improve colonic histology by decreasing caspase-3 in colons (Nooh and Nour-Eldien, 2016).
In 2021, Elmaksoud et al. demonstrated that Hydroxytyrosol $(50 \mathrm{mg} / \mathrm{kg})$, one of the main alcoholic compounds of the olive leaves extract, down-regulated the expression of bax and upregulated that of bcl-2 in the colons of acetic acid-induced colitis rats (Elmaksoud et al., 2021). Tanshinol, a bioactive ingredient in DanShen [Lamiaceae: Salvia miltiorrhiza Bunge], was reported by Zhu et al. to alleviate apoptosis in UC model cells through promoting very low-density lipoprotein receptor expression (Zhu et al., 2021).

\section{CONCLUSIONS AND PERSPECTIVES}

Natural products refer to a wide range of bioactive extracts or isolated metabolites from natural materials. Their bioactivities are currently of great interest in many research fields (Ekiert and Szopa, 2020) and may yield promising pharmacological approaches for the prevention and treatment of UC due to their multiple regulatory effects with few adverse effects (Nascimento et al., 2020). In recent years, converging lines of evidence have demonstrated that apoptosis of IECs is highly associated with the occurrence and development of UC. This review reported that multiple natural products have antiapoptotic activities in vitro and in vivo to protect intestinal epithelial cells against apoptosis in UC. Furthermore, their potential mechanisms are closely associated with the regulation of multiple apoptosis-related signaling pathways, including death-receptor mediated pathway, mitochondrialdependent pathway, ERS-mediated pathway, MAPK-mediated pathway, NF- $\mathrm{B}$ mediated pathway, P13k/Akt pathway, and other reported pathways such as JAK/STAT3 and NLRP3/ ASC/Caspase-1. Thus, it is rational to presume that natural products may yield promising therapeutic agents to treat UC patients by modulating apoptosis of IECs.

Although many natural products have been demonstrated to be the potential candidates for UC treatment by targeting intestinal epithelial apoptosis, more sophisticated works in preclinical and clinical investigations need to be performed to research and develop effective pharmacotherapies. First, studies on the pharmacokinetics and pharmacodynamics of these natural agents are insufficient. Recently, with the increasing attention drawn on natural products, great attention has been paid to metabolism and pharmacokinetics research (Zeng et al., 2017), laying a foundation for subsequent research of toxicology and medication safety. Second, systematic evaluation for the toxicity and safety of natural products remains scarce. Though these natural plants and botanical drugs have been wildly used for thousands of years, well-designed studies for critical evaluation of safety are imperative for developing novel and effective pharmacotherapeutic agents. More concerns need to be paid on the potential toxicity and adverse effects of natural products (Wu et al., 2016; Byard et al., 2017; Liu et al., 2020). Third, current research of natural products targeting apoptosis in UC has primarily focused on their in vivo and in vitro effects and mechanisms. Well-designed clinical trials with high methodological quality are urgently needed for further verification of these natural products. Lastly, the exploration of 
mechanisms of some natural products is still in the preliminary stage, and the specific targets and signaling pathways require further elucidation. In addition, some of the above-mentioned natural products, such as baicalin, Indirubin, and Paeoniflorin, are reported to modulate apoptosis through multiple pathways, and their underlying interactions or crosstalk with the core-target network of UC are worthy of further exploration.

In summary, we expect that this review will provide helpful information to understand the effects of natural products and their pharmacological mechanisms in regulating intestinal epithelial apoptosis of UC. These natural extracts and isolated metabolites are of potential value in clinical UC management. We also expect more researchers and clinicians to pay close attention to this field and conduct more relevant studies and trials.

\section{REFERENCES}

Adams, S. M., and Bornemann, P. H. (2013). Ulcerative Colitis. Am. Fam. Physician 87 (10), 699-705.

Ananthakrishnan, A. N. (2015). Epidemiology and Risk Factors for IBD. Nat. Rev. Gastroenterol. Hepatol. 12 (4), 205-217. doi:10.1038/nrgastro.2015.34

Araki, Y., Mukaisyo, K., Sugihara, H., Fujiyama, Y., and Hattori, T. (2010). Increased Apoptosis and Decreased Proliferation of Colonic Epithelium in Dextran Sulfate Sodium-Induced Colitis in Mice. Oncol. Rep. 24 (4), 869-874. doi:10.3892/or.2010.869

Aranha, M. M., Borralho, P. M., Ravasco, P., Moreira da Silva, I. B., Correia, L., Fernandes, A., et al. (2007). NF-kappaB and Apoptosis in Colorectal Tumourigenesis. Eur. J. Clin. Invest. 37 (5), 416-424. doi:10.1111/j.13652362.2007.01801.x

Baldwin, A. S. (2012). Regulation of Cell Death and Autophagy by IKK and NFkappaB: Critical Mechanisms in Immune Function and Cancer. Immunol. Rev. 246 (1), 327-345. doi:10.1111/j.1600-065X.2012.01095.x

Bopanna, S., Ananthakrishnan, A. N., Kedia, S., Yajnik, V., and Ahuja, V. (2017). Risk of Colorectal Cancer in Asian Patients with Ulcerative Colitis: a Systematic Review and Meta-Analysis. Lancet Gastroenterol. Hepatol. 2 (4), 269-276. doi:10.1016/S2468-1253(17)30004-3

Burri, E., Maillard, M. H., Schoepfer, A. M., Seibold, F., Van Assche, G., Riviere, P., et al. (2020). Treatment Algorithm for Mild and Moderate-To-Severe Ulcerative Colitis: An Update. Digestion 101 (Suppl. 1), 2-15. doi:10.1159/ 000504092

Byard, R. W., Musgrave, I., Maker, G., and Bunce, M. (2017). What Risks Do Herbal Products Pose to the Australian Community? Med. J. Aust. 206 (2), 86-90. doi:10.5694/mja16.00614

Cao, S. S. (2016). Epithelial ER Stress in Crohn's Disease and Ulcerative Colitis. Inflamm. Bowel Dis. 22 (4), 984-993. doi:10.1097/MIB.0000000000000660

Cao, S. Y., Ye, S. J., Wang, W. W., Wang, B., Zhang, T., and Pu, Y. Q. (2019). Progress in Active Compounds Effective on Ulcerative Colitis from Chinese Medicines. Chin. J. Nat. Med. 17 (2), 81-102. doi:10.1016/S1875-5364(19) 30012-3

Chen, Q., Duan, X., Fan, H., Xu, M., Tang, Q., Zhang, L., et al. (2017). Oxymatrine Protects against DSS-Induced Colitis via Inhibiting the PI3K/AKT Signaling Pathway. Int. Immunopharmacol 53, 149-157. doi:10.1016/j.intimp.2017. 10.025

Chen, Z., Zheng, Y., Yang, J., and Lu, Y. (2021). Effect of Chushi Jianpi Decoction on IL-10/JAK1/STAT3 Pathway and Apoptosis of Intestinal Epithelial Cells in Mice with Ulcerative Colitis (China). Guid J. Tradit Chin. Med. Pharm. 27 (5), 13-17. doi:10.13862/j.cnki.cn43-1446/r.2021.05.001

Conrad, K., Roggenbuck, D., and Laass, M. W. (2014). Diagnosis and Classification of Ulcerative Colitis. Autoimmun. Rev. 13 (4-5), 463-466. doi:10.1016/j.autrev. 2014.01.028

Dong, J. Y., Xia, K. J., Liang, W., Liu, L. L., Yang, F., Fang, X. S., et al. (2021). Ginsenoside Rb1 Alleviates Colitis in Mice via Activation of Endoplasmic

\section{AUTHOR CONTRIBUTIONS}

All authors contributed substantially to the article. YL conceived and designed this paper. CHL, YWZ, YLW, and XGH summarized and analyzed the data. CHL and YWZ drafted, revised, and edited the paper.

\section{FUNDING}

This research was supported by the project of State Administration of Traditional Chinese Medicine of the People's Republic of China (No. GZY-KJS-2020-024) and the Major Research and Development Project of Sichuan Provincial Science and Technology Department (No. 22ZDYF2011).

Reticulum-Resident E3 Ubiquitin Ligase Hrd1 Signaling Pathway. Acta Pharmacol. Sin 42 (9), 1461-1471. doi:10.1038/s41401-020-00561-9

Du, L., and Ha, C. (2020). Epidemiology and Pathogenesis of Ulcerative Colitis. Gastroenterol. Clin. North. Am. 49 (4), 643-654. doi:10.1016/j.gtc.2020.07.005

Eissa, N., Hussein, H., Diarra, A., Elgazzar, O., Gounni, A. S., Bernstein, C. N., et al. (2019). Semaphorin 3E Regulates Apoptosis in the Intestinal Epithelium during the Development of Colitis. Biochem. Pharmacol. 166, 264-273. doi:10.1016/j. bcp.2019.05.029

Ekiert, H. M., and Szopa, A. (2020). Biological Activities of Natural Products. Molecules 25 (23). doi:10.3390/molecules25235769

Elmaksoud, H. A. A., Motawea, M. H., Desoky, A. A., Elharrif, M. G., and Ibrahimi, A. (2021). Hydroxytyrosol Alleviate Intestinal Inflammation, Oxidative Stress and Apoptosis Resulted in Ulcerative Colitis. Biomed. Pharmacother. 142, 112073. doi:10.1016/j.biopha.2021.112073

Elmore, S. (2007). Apoptosis: a Review of Programmed Cell Death. Toxicol. Pathol. 35 (4), 495-516. doi:10.1080/01926230701320337

Estaquier, J., Vallette, F., Vayssiere, J. L., and Mignotte, B. (2012). The Mitochondrial Pathways of Apoptosis. Adv. Exp. Med. Biol. 942, 157-183. doi:10.1007/978-94-007-2869-1_7

Fan, H., Li, X., Duan, X., and Shi, T. (2020). Cell Apoptosis in the Etiopathogenesis of Ulcerative Colitis. Chin. J. Integrated Traditional West. Med. Digestion 10 (3), 189-190.

Fan, T. J., Han, L. H., Cong, R. S., and Liang, J. (2005). Caspase Family Proteases and Apoptosis. Acta Biochim. Biophys. Sin (Shanghai). 37 (11), 719-727. doi:10. 1111/j.1745-7270.2005.00108.x

Feng, P., Tao, Z., and Jianyong, C. (2010). The Polysaccharide of Portulaca Oleracea Interfered $\mathrm{W}$ Ith the Express Ion of Caspase- 3 and Caspase- 8 in a Rat Expermiental Model of TNBS-Induced Colitis. CHINESE ARCHIVES TRADITIONAL CHINESE MEDICINE 28 (8), 1639-1641. doi:10.13193/j. archtcm.2010.08.73.panf.074

Franke, T. F., Hornik, C. P., Segev, L., Shostak, G. A., and Sugimoto, C. (2003). PI3K/Akt and Apoptosis: Size Matters. Oncogene 22 (56), 8983-8998. doi:10. 1038/sj.onc. 1207115

Fresno Vara, J. A., Casado, E., de Castro, J., Cejas, P., Belda-Iniesta, C., and Gonzalez-Baron, M. (2004). PI3K/Akt Signalling Pathway and Cancer. Cancer Treat. Rev. 30 (2), 193-204. doi:10.1016/j.ctrv.2003.07.007

Gao, W., Wang, C., Yu, L., Sheng, T., Wu, Z., Wang, X., et al. (2019). Chlorogenic Acid Attenuates Dextran Sodium Sulfate-Induced Ulcerative Colitis in Mice through MAPK/ERK/JNK Pathway. Biomed. Res. Int. 2019, 6769789. doi:10. $1155 / 2019 / 6769789$

Gao, W., Zhang, L., Wang, X., Yu, L., Wang, C., and Gong, Y. (2018). The Combination of Indirubin and Isatin Attenuates Dextran Sodium Sulfate Induced Ulcerative Colitis in Mice. Biochem. Cel Biol 96 (5), 636-645. doi:10.1139/bcb-2018-0041

Gu, B. H., Minh, N. V., Lee, S. H., Lim, S. W., Lee, Y. M., Lee, K. S., et al. (2010). Deoxyschisandrin Inhibits H2O2-Induced Apoptotic Cell Death in Intestinal Epithelial Cells through Nuclear Factor-kappaB. Int. J. Mol. Med. 26 (3), 401-406. 
Gu, P., Zhu, L., Liu, Y., Zhang, L., Liu, J., and Shen, H. (2017). Protective Effects of Paeoniflorin on TNBS-Induced Ulcerative Colitis through Inhibiting NFkappaB Pathway and Apoptosis in Mice. Int. Immunopharmacol 50, 152-160. doi:10.1016/j.intimp.2017.06.022

Hagiwara, C., Tanaka, M., and Kudo, H. (2002). Increase in Colorectal Epithelial Apoptotic Cells in Patients with Ulcerative Colitis Ultimately Requiring Surgery. J. Gastroenterol. Hepatol. 17 (7), 758-764. doi:10.1046/j.1440-1746. 2002.02791.x

Hassanshahi, N., Masoumi, S. J., Mehrabani, D., Hashemi, S. S., and Zare, M. (2020). The Healing Effect of Aloe Vera Gel on Acetic Acid-Induced Ulcerative Colitis in Rat. Middle East. J. Dig. Dis. 12 (3), 154-161. doi:10.34172/mejdd. 2020.177

Heinrich, M., Appendino, G., Efferth, T., Furst, R., Izzo, A. A., Kayser, O., et al. (2020). Best Practice in Research - Overcoming Common Challenges in Phytopharmacological Research. J. Ethnopharmacol 246, 112230. doi:10. 1016/j.jep.2019.112230

Helal, M. G., and Abd Elhameed, A. G. (2021). Graviola Mitigates Acetic AcidInduced Ulcerative Colitis in Rats: Insight on Apoptosis and Wnt/Hh Signaling Crosstalk. Environ. Sci. Pollut. Res. Int. 28 (23), 29615-29628. doi:10.1007/ s11356-021-12716-0

Hetz, C. (2012). The Unfolded Protein Response: Controlling Cell Fate Decisions under ER Stress and beyond. Nat. Rev. Mol. Cel Biol 13 (2), 89-102. doi:10.1038/ nrm3270

Heyninck, K., and Beyaert, R. (2001). Crosstalk between NF-kappaB-Activating and Apoptosis-Inducing Proteins of the TNF-Receptor Complex. Mol. Cel Biol Res Commun 4 (5), 259-265. doi:10.1006/mcbr.2001.0295

Huang, X. L., Xu, J., Zhang, X. H., Qiu, B. Y., Peng, L., Zhang, M., et al. (2011). PI3K/Akt Signaling Pathway Is Involved in the Pathogenesis of Ulcerative Colitis. Inflamm. Res. 60 (8), 727-734. doi:10.1007/s00011-011-0325-6

Iwamoto, M., Koji, T., Makiyama, K., Kobayashi, N., and Nakane, P. K. (1996). Apoptosis of Crypt Epithelial Cells in Ulcerative Colitis. J. Pathol. 180 (2), 152-159. doi:10.1002/(SICI)1096-9896(199610)180:2<152:AID-PATH649>3. $0 . \mathrm{CO} ; 2-\mathrm{Y}$

Jeong, S. Y., and Seol, D. W. (2008). The Role of Mitochondria in Apoptosis. BMB Rep. 41 (1), 11-22. doi:10.5483/bmbrep.2008.41.1.011

Kantari, C., and Walczak, H. (2011). Caspase-8 and Bid: Caught in the Act between Death Receptors and Mitochondria. Biochim. Biophys. Acta 1813 (4), 558-563. doi:10.1016/j.bbamcr.2011.01.026

Ke, F., Yadav, P. K., and Ju, L. Z. (2012). Herbal Medicine in the Treatment of Ulcerative Colitis. Saudi J. Gastroenterol. 18 (1), 3-10. doi:10.4103/1319-3767. 91726

Kim, M., Chung, K. S., Hwang, S. J., Yoon, Y. S., Jang, Y. P., Lee, J. K., et al. (2020). Protective Effect of Cicer Arietinum L. (Chickpea) Ethanol Extract in the Dextran Sulfate Sodium-Induced Mouse Model of Ulcerative Colitis. Nutrients 12 (2). doi:10.3390/nu12020456

Kong, R., Luo, H., Wang, N., Li, J., Xu, S., Chen, K., et al. (2018). Portulaca Extract Attenuates Development of Dextran Sulfate Sodium Induced Colitis in Mice through Activation of PPARgamma. PPAR Res. 2018, 6079101. doi:10.1155/ 2018/6079101

Lanzhen, L., Shuangyan, L., Ye, W., and Xiaoli, S. (2020). Effects of Paeoniflorin on Expressions of Beclin1 and Bcl-2 in Colon Tissue of Rats with DSS-Induced Chronic Ulcerative Colitis (China). Chin. J. Traditional Med. Sci. Technology 27 (6), 885-889.

Li, Q., Chen, M., Liu, H., Yang, L., Yang, T., and He, G. (2014). The Dual Role of ERK Signaling in the Apoptosis of Neurons. Front. Biosci. (Landmark Ed. 19, 1411-1417. doi:10.2741/4291

Li, Q., Li, K., Hu, T., Liu, F., Liao, S., and Zou, Y. (2021). 6,7-Dihydroxy-2,4Dimethoxyphenanthrene from Chinese Yam Peels Alleviates DSS-Induced Intestinal Mucosal Injury in Mice via Modulation of the NF-kappaB/COX-2 Signaling Pathway. J. Agric. Food Chem. 69 (16), 4720-4731. doi:10.1021/acs. jafc. $1 \mathrm{c} 00487$

Lin, H., Honglang, L., Weifeng, L., Junmin, C., Jiantao, Y., and Junjing, G. (2017). The Mechanism of Alopolysaccharide Protecting Ulceralive Colitis. Biomed. Pharmacother. 88, 145-150. doi:10.1016/j.biopha.2016.11.138

Lin, J. C., Wu, J. Q., Wang, F., Tang, F. Y., Sun, J., Xu, B., et al. (2019). QingBai Decoction Regulates Intestinal Permeability of Dextran Sulphate SodiumInduced Colitis through the Modulation of Notch and NF-kappaB Signalling. Cell Prolif 52 (2), e12547. doi:10.1111/cpr.12547
Liu, D., Huang, X., Cheng, S., Tong, W., Wan, P., Guan, Y., et al. (2011). Regulation of Sishen Wan on Bax/Bcl-2 mRNA, Fas/FasL in Colonic Tissue from Rats with Colitis (China). Zhongguo Zhong Yao Za Zhi 36 (24), 3484-3488.

Liu, D., Zhang, M., and Yin, H. (2013). Signaling Pathways Involved in Endoplasmic Reticulum Stress-Induced Neuronal Apoptosis. Int. J. Neurosci. 123 (3), 155-162. doi:10.3109/00207454.2012.746974

Liu, F., Bardhan, K., Yang, D., Thangaraju, M., Ganapathy, V., Waller, J. L., et al. (2012). NF-kappaB Directly Regulates Fas Transcription to Modulate FasMediated Apoptosis and Tumor Suppression. J. Biol. Chem. 287 (30), 25530-25540. doi:10.1074/jbc.M112.356279

Liu, R., Li, X., Huang, N., Fan, M., and Sun, R. (2020). Toxicity of Traditional Chinese Medicine Herbal and mineral Products. Adv. Pharmacol. 87, 301-346. doi:10.1016/bs.apha.2019.08.001

Liu, X., and Wang, J. M. (2011). Iridoid Glycosides Fraction of Folium Syringae Leaves Modulates NF-kappaB Signal Pathway and Intestinal Epithelial Cells Apoptosis in Experimental Colitis. PLoS One 6 (9), e24740. doi:10.1371/journal. pone. 0024740

Lv, T., Shen, L., Yang, L., Diao, W., Yang, Z., Zhang, Y., et al. (2018). Polydatin Ameliorates Dextran Sulfate Sodium-Induced Colitis by Decreasing Oxidative Stress and Apoptosis Partially via Sonic Hedgehog Signaling Pathway. Int. Immunopharmacol 64, 256-263. doi:10.1016/j.intimp.2018. 09.009

Mahmoud, T. N., El-Maadawy, W. H., Kandil, Z. A., Khalil, H., El-Fiky, N. M., and El Alfy, T. (2021). Canna X Generalis L.H. Bailey Rhizome Extract Ameliorates Dextran Sulfate Sodium-Induced Colitis via Modulating Intestinal Mucosal Dysfunction, Oxidative Stress, Inflammation, and TLR4/NF-B and NLRP3 Inflammasome Pathways. J. Ethnopharmacol 269, 113670. doi:10.1016/j.jep. 2020.113670

Miao, F., Shan, C., Ma, T., Geng, S., and Ning, D. (2021). Walnut Oil Alleviates DSS-Induced Colitis in Mice by Inhibiting NLRP3 Inflammasome Activation and Regulating Gut Microbiota. Microb. Pathog. 154, 104866. doi:10.1016/j. micpath.2021.104866

Motawea, M. H., Abd Elmaksoud, H. A., Elharrif, M. G., Desoky, A. A. E., and Ibrahimi, A. (2020). Evaluation of Anti-inflammatory and Antioxidant Profile of Oleuropein in Experimentally Induced Ulcerative Colitis. Int. J. Mol. Cel Med 9 (3), 224-233. doi:10.22088/IJMCM.BUMS.9.3.224

Nan, B., Ye, W., Rui, W., Li-ting, S., and Yan-qiu, F. (2019). Tripterygium Glycosides Attenuate Intestinal Mucosal Apoptosis and Inflammation in Rats with Ulcerative Colitis through JAK2/STAT3 Signaling Pathway (China). Mod. Digestion \& Intervention 24 (5), 466-470.

Nascimento, R. P. D., Machado, A., Galvez, J., Cazarin, C. B. B., and Marostica Junior, M. R. (2020). Ulcerative Colitis: Gut Microbiota, Immunopathogenesis and Application of Natural Products in Animal Models. Life Sci. 258, 118129. doi:10.1016/j.lfs.2020.118129

Ng, S. C., Shi, H. Y., Hamidi, N., Underwood, F. E., Tang, W., Benchimol, E. I., et al. (2017). Worldwide Incidence and Prevalence of Inflammatory Bowel Disease in the 21st century: a Systematic Review of Population-Based Studies. Lancet 390 (10114), 2769-2778. doi:10.1016/S0140-6736(17)32448-0

Nooh, H. Z., and Nour-Eldien, N. M. (2016). The Dual Anti-inflammatory and Antioxidant Activities of Natural Honey Promote Cell Proliferation and Neural Regeneration in a Rat Model of Colitis. Acta Histochem. 118 (6), 588-595. doi:10.1016/j.acthis.2016.06.006

Polat, F. R., and Karaboga, I. (2019). Immunohistochemical Examination of Antiinflammatory and Anti-apoptotic Effects of Hesperetin on Trinitrobenzene Sulfonic Acid Induced Colitis in Rats. Biotech. Histochem. 94 (3), 151-158. doi:10.1080/10520295.2018.1530454

Pugliese, D., Felice, C., Papa, A., Gasbarrini, A., Rapaccini, G. L., Guidi, L., et al. (2017). Anti TNF-Alpha Therapy for Ulcerative Colitis: Current Status and Prospects for the Future. Expert Rev. Clin. Immunol. 13 (3), 223-233. doi:10. 1080/1744666X.2017.1243468

Qiu, W., Wu, B., Wang, X., Buchanan, M. E., Regueiro, M. D., Hartman, D. J., et al. (2011). PUMA-mediated Intestinal Epithelial Apoptosis Contributes to Ulcerative Colitis in Humans and Mice. J. Clin. Invest. 121 (5), 1722-1732. doi:10.1172/JCI42917

Rapa, S. F., Di Paola, R., Cordaro, M., Siracusa, R., D’Amico, R., Fusco, R., et al. (2021). Plumericin Protects against Experimental Inflammatory Bowel Disease by Restoring Intestinal Barrier Function and Reducing Apoptosis. Biomedicines 9 (1). doi:10.3390/biomedicines 9010067 
Rioux, K. (2008). What Is the Prognosis of Ulcerative Colitis? Inflamm. Bowel Dis. 14 (Suppl. 2), S52-S53. doi:10.1002/ibd.20574

Santana, M. T., Cercato, L. M., Oliveira, J. P., and Camargo, E. A. (2017). Medicinal Plants in the Treatment of Colitis: Evidence from Preclinical Studies. Planta Med. 83 (7), 588-614. doi:10.1055/s-0043-104933

Schulzke, J. D., Bojarski, C., Zeissig, S., Heller, F., Gitter, A. H., and Fromm, M. (2006). Disrupted Barrier Function through Epithelial Cell Apoptosis. Ann. N. Y Acad. Sci. 1072, 288-299. doi:10.1196/annals.1326.027

Schulzke, J. D., Ploeger, S., Amasheh, M., Fromm, A., Zeissig, S., Troeger, H., et al. (2009). Epithelial Tight Junctions in Intestinal Inflammation. Ann. N. Y Acad. Sci. 1165, 294-300. doi:10.1111/j.1749-6632.2009.04062.x

Seidelin, J. B., and Nielsen, O. H. (2009). Epithelial Apoptosis: Cause or Consequence of Ulcerative Colitis? Scand. J. Gastroenterol. 44 (12), 1429-1434. doi:10.3109/00365520903301212

Sharma, A., Tirpude, N. V., Kulurkar, P. M., Sharma, R., and Padwad, Y. (2020). Berberis Lycium Fruit Extract Attenuates Oxi-Inflammatory Stress and Promotes Mucosal Healing by Mitigating NF-kappaB/c-Jun/MAPKs Signalling and Augmenting Splenic Treg Proliferation in a Murine Model of Dextran Sulphate Sodium-Induced Ulcerative Colitis. Eur. J. Nutr. 59 (6), 2663-2681. doi:10.1007/s00394-019-02114-1

Shen, J., Cheng, J., Zhu, S., Zhao, J., Ye, Q., Xu, Y., et al. (2019). Regulating Effect of Baicalin on IKK/IKB/NF-kB Signaling Pathway and Apoptosis-Related Proteins in Rats with Ulcerative Colitis. Int. Immunopharmacol 73, 193-200. doi:10.1016/j.intimp.2019.04.052

Shi, L., Dai, Y., Jia, B., Han, Y., Guo, Y., Xie, T., et al. (2019). The Inhibitory Effects of Qingchang Wenzhong Granule on the Interactive Network of Inflammation, Oxidative Stress, and Apoptosis in Rats with Dextran Sulfate Sodium-Induced Colitis. J. Cel Biochem 120 (6), 9979-9991. doi:10.1002/jcb.28280

Shuguang, Y., Yi, H., and Jingtao, L. (2016). Effect of Wumei Pill on Apoptosis of Colonic Epithelial Cells in Rats with Ulcerative Colitis (China). J. Basic Chin. Med. 22 (6), 771-773.

Song, C., Chen, J., Li, X., Yang, R., Cao, X., Zhou, L., et al. (2021). Limonin Ameliorates Dextran Sulfate Sodium-Induced Chronic Colitis in Mice by Inhibiting PERK-ATF4-CHOP Pathway of ER Stress and NF-kappaB Signaling. Int. Immunopharmacol 90, 107161. doi:10.1016/j.intimp.2020.107161

Soubh, A. A., Abdallah, D. M., and El-Abhar, H. S. (2015). Geraniol Ameliorates TNBS-Induced Colitis: Involvement of Wnt/beta-Catenin, p38MAPK, NFkappaB, and PPARgamma Signaling Pathways. Life Sci. 136, 142-150. doi:10.1016/j.lfs.2015.07.004

Sun, Y., Liu, W. Z., Liu, T., Feng, X., Yang, N., and Zhou, H. F. (2015). Signaling Pathway of MAPK/ERK in Cell Proliferation, Differentiation, Migration, Senescence and Apoptosis. J. Recept Signal. Transduct Res. 35 (6), 600-604. doi:10.3109/10799893.2015.1030412

Tan, Y., Dourdin, N., Wu, C., De Veyra, T., Elce, J. S., and Greer, P. A. (2006). Ubiquitous Calpains Promote Caspase-12 and JNK Activation during Endoplasmic Reticulum Stress-Induced Apoptosis. J. Biol. Chem. 281 (23), 16016-16024. doi:10.1074/jbc.M601299200

Taya, S., Kakehashi, A., Wongpoomchai, R., Gi, M., Ishii, N., and Wanibuchi, H. (2016). Preventive Effects of Spirogyra Neglecta and a Polysaccharide Extract against Dextran Sodium Sulfate Induced Colitis in Mice. Asian Pac. J. Cancer Prev. 17 (4), 2235-2245. doi:10.7314/apjcp.2016.17.4.2235

Thorburn, A. (2004). Death Receptor-Induced Cell Killing. Cell Signal 16 (2), 139-144. doi:10.1016/j.cellsig.2003.08.007

Topcu-Tarladacalisir, Y., Akpolat, M., Uz, Y. H., Kizilay, G., Sapmaz-Metin, M., Cerkezkayabekir, A., et al. (2013). Effects of Curcumin on Apoptosis and Oxidoinflammatory Regulation in a Rat Model of Acetic Acid-Induced Colitis: the Roles of C-Jun N-Terminal Kinase and P38 Mitogen-Activated Protein Kinase. J. Med. Food 16 (4), 296-305. doi:10.1089/jmf.2012.2550

Triantafyllidi, A., Xanthos, T., Papalois, A., and Triantafillidis, J. K. (2015). Herbal and Plant Therapy in Patients with Inflammatory Bowel Disease. Ann. Gastroenterol. 28 (2), 210-220.

Valmiki, M. G., and Ramos, J. W. (2009). Death Effector Domain-Containing Proteins. Cell Mol Life Sci 66 (5), 814-830. doi:10.1007/s00018-008-8489-0

Vanamee, E. S., and Faustman, D. L. (2018). Structural Principles of Tumor Necrosis Factor Superfamily Signaling. Sci. Signal. 11 (511). doi:10.1126/scisignal.aao4910

Verstege, M. I., te Velde, A. A., and Hommes, D. W. (2006). Apoptosis as a Therapeutic Paradigm in Inflammatory Bowel Diseases. Acta Gastroenterol. Belg. 69 (4), 406-412.
Vivanco, I., and Sawyers, C. L. (2002). The Phosphatidylinositol 3-Kinase AKT Pathway in Human Cancer. Nat. Rev. Cancer 2 (7), 489-501. doi:10.1038/nrc839

Vukelic, I., Detel, D., Baticic, L., Potocnjak, I., and Domitrovic, R. (2020). Luteolin Ameliorates Experimental Colitis in Mice through ERK-Mediated Suppression of Inflammation, Apoptosis and Autophagy. Food Chem. Toxicol. 145, 111680. doi:10.1016/j.fct.2020.111680

Wan, P., Chen, H., Guo, Y., and Bai, A. P. (2014). Advances in Treatment of Ulcerative Colitis with Herbs: from Bench to Bedside. World J. Gastroenterol. 20 (39), 14099-14104. doi:10.3748/wjg.v20.i39.14099

Wang, X., Li, D., Zhang, Y., Wu, S., and Tang, F. (2018). Costus Root Granules Improve Ulcerative Colitis through Regulation of TGF-Beta Mediation of the PI3K/AKT Signaling Pathway. Exp. Ther. Med. 15 (5), 4477-4484. doi:10.3892/ etm.2018.5946

Wang, Y., Liu, J., Huang, Z., Li, Y., Liang, Y., Luo, C., et al. (2021). Coptisine Ameliorates DSS-Induced Ulcerative Colitis via Improving Intestinal Barrier Dysfunction and Suppressing Inflammatory Response. Eur. J. Pharmacol. 896, 173912. doi:10.1016/j.ejphar.2021.173912

Wehkamp, J., and Stange, E. F. (2018). Recent Advances and Emerging Therapies in the Non-surgical Management of Ulcerative Colitis. F1000Res 7. doi:10. 12688/f1000research.15159.1

Weijie, P., Lianfeng, Z., and Liang, L. (2019). A Study on Effect of Astragalus Polysaccharide on Rats with Ulcerative Colitis and its Mechanism (China). JOURNAL NEW CHINESE MEDICINE 51 (9), 13-16. doi:10.13457/j.cnki. jncm.2019.09.004

Wenqiang, M., Kun, Y., Xin, T., and Linxuan, S. (2019). The Therapeutic Effect and Mechanism of Indigo on Dextran Sulfate Sodium Induced Ulcerative Colitis in Mice (China). Prog. Anatomical Sci. 25 (2), 146-149. doi:10.16695/j.cnki.10062947.2019.02.010

Wu, H., Zhong, R. L., Xia, Z., Huang, H. C., Zhong, Q. X., Feng, L., et al. (2016). Research Progress on Potential Liver Toxic Components in Traditional Chinese Medicine. Zhongguo Zhong Yao Za Zhi 41 (17), 3209-3217. doi:10.4268/ cjcmm20161715

Xian-juan, Y., Yin, F., Jian, W., and al, e. (2020). Protective Effect of Compatibility of Coptidis Rhizoma and Magnoliae Officinalis Cortex on Rat Model of Ulcerative Colitis and Influence of Apoptosis (China). Chin. J. Exp. Traditional Med. Formulae 26 (23), 83-91. doi:10.13422/j.cnki.syfjx.20202238

Xiao, H. T., Lin, C. Y., Ho, D. H., Peng, J., Chen, Y., Tsang, S. W., et al. (2013). Inhibitory Effect of the Gallotannin Corilagin on Dextran Sulfate SodiumInduced Murine Ulcerative Colitis. J. Nat. Prod. 76 (11), 2120-2125. doi:10. $1021 / \mathrm{np} 4006772$

Xiaobin, Z., and Xiaodong, X. (2014). Effects of Laggera Alata Flavone on Ulcerative Colitis and its Mechanism (China). CHINESE ARCHIVES TRADITIONAL CHINESE MEDICINE 32 (12), 3028-3030. doi:10.13193/j. issn.1673-7717.2014.12.069

Yan, D., and Jingen, L. (2016). The Effects of Aucklandia and Coptis Pills on Cellular Apoptosis and the Expression of Bcl-2 and Bax mRNA in Model Rats with Ulcerative Colitis (China). West China Med. J. 31 (6), 1046-1051. doi:10. 7507/1002-0179.201600281

Yan, J., Ouyang, Q., Chen, D., et al. (2001). The Role of Fas/FasL-Mediated Colonic Epithelium Apoptosis in Ulcerative Colitis (China). Chin. J. Dig. 21 (7), 397-399.

Yan, S., and Bin, L. (2020). Study of Glycyrrhizin Regulating Caspase-12 Apoptosis Signaling Pathway of Endoplasmic Reticulum Stress to Alleviate the Colonic Inflammatory Response in Ulcerative Colitis. CJTCMP 35 (8), 3872-3877.

Yan, S., Si-yi, N., Hua-jun, Z., Si, L., and Bin, L. (2021). Mechanisms of Gancao Xiexin Decoction on Protecting Intestinal Mucosal Barrier by Regulating PERK-elF2a-CHOP Signaling Pathway (China). China J. Traditional Chin. Med. Pharm. 36 (5), 2657-2663.

Yan, S., Yingchao, L., Zhangliu, W., Xianli, R., Si, L., Siyi, N., et al. (2020). Effect of Berberine from Coptis Chinensis on Apoptosis of Intestinal Epithelial Cells in a Mouse Model of Ulcerative Colitis: Role of Endoplasmic Reticulum Stress. Evid. Based Complement. Alternat Med. 2020, 3784671. doi:10.1155/2020/3784671

Yan, S., Zhangliu, W., Huajun, Z., and al, e. (2018). Effects of Berberine on Apoptosis of Intestinal Epithelial Cells in the Ulcerative Colitis Mice (China). Zhejiang JITCWM 28 (12), 992-996.

Yang, M., Liu, W., Tu, H., Li, L., and Fei, S. (2020). Protective Effect of Crocin in Ulcerative Colitis Rats and its Related Mechanism (China). Chin. J. Tissue Eng. Res. 24 (29), 4673-4679. doi:10.3969/j.issn.2095-4344.2817 
Yao, J., Cao, X., Zhang, R., Li, Y. X., Xu, Z. L., Zhang, D. G., et al. (2016). Protective Effect of Baicalin against Experimental Colitis via Suppression of Oxidant Stress and Apoptosis. Pharmacogn Mag. 12 (47), 225-234. doi:10.4103/0973-1296. 186342

Yi, H., Shuguang, Y., and Xiaolong, W. (2016). Study on Effect of Wumei Pill in Colonic Epithelial Cell Apoptosis and Expression of Bcl-2/Bax of Ulcerative Colitis in Rats (China). CHINESE ARCHIVES TRADITIONAL CHINESE MEDICINE 34 (1), 149-151.

Yin, S., Li, L., Tao, Y., Yu, J., Wei, S., Liu, M., et al. (2021). The Inhibitory Effect of Artesunate on Excessive Endoplasmic Reticulum Stress Alleviates Experimental Colitis in Mice. Front. Pharmacol. 12, 629798. doi:10.3389/ fphar.2021.629798

Yin, S., Yang, H., Tao, Y., Wei, S., Li, L., Liu, M., et al. (2020). Artesunate Ameliorates DSS-Induced Ulcerative Colitis by Protecting Intestinal Barrier and Inhibiting Inflammatory Response. Inflammation 43 (2), 765-776. doi:10. 1007/s10753-019-01164-1

Yong, Z., Wen-hua, S., Zheng-xia, Z., Luan, H., Yan-li, L., and Qiong-ming, X. (2020). Experimental Study on Pharmacological Mechanism ofAnemoside B4 in Ulcerative Colitis Induced by Acetic Acid in Rats (China). Anti Infect. Pharm. 17 (10), 1405-1410. doi:10.13493/j.issn.1672-7878.2020.10-002

Youle, R. J., and Strasser, A. (2008). The BCL-2 Protein Family: Opposing Activities that Mediate Cell Death. Nat. Rev. Mol. Cel Biol 9 (1), 47-59. doi:10.1038/ nrm2308

Yu, S., and Qian, H. (2021). Deoxyschizandrin Treats Mice with Ulcerative Colitis Possibly via the TLR4/NF-kappaB Signaling Pathway. Am. J. Transl Res. 13 (4), 3856-3863.

Yu, X., Cai, Y., Zhou, S., Cheng, H., Zhou, W., and Li, X. (2021). Therapeutic Effect of Hyperoside in Rats with Ulcerative Colitis and its Mechanism (China). IMMUNOLOGICAL JOURNAL 37 (5), 417-424. doi:10.13431/j.cnki.immunol. j.20210058

Yu, Y. R., and Rodriguez, J. R. (2017). Clinical Presentation of Crohn's, Ulcerative Colitis, and Indeterminate Colitis: Symptoms, Extraintestinal Manifestations, and Disease Phenotypes. Semin. Pediatr. Surg. 26 (6), 349-355. doi:10.1053/j. sempedsurg.2017.10.003

Yue, J., and Lopez, J. M. (2020). Understanding MAPK Signaling Pathways in Apoptosis. Int. J. Mol. Sci. 21 (7). doi:10.3390/ijms21072346

Yukawa, M., Iizuka, M., Horie, Y., Yoneyama, K., Shirasaka, T., Itou, H., et al. (2002). Systemic and Local Evidence of Increased Fas-Mediated Apoptosis in Ulcerative Colitis. Int. J. Colorectal Dis. 17 (2), 70-76. doi:10.1007/ s003840100340

Zeng, M., Yang, L., He, D., Li, Y., Shi, M., and Zhang, J. (2017). Metabolic Pathways and Pharmacokinetics of Natural Medicines with Low Permeability. Drug Metab. Rev. 49 (4), 464-476. doi:10.1080/03602532.2017.1377222

Zhang, J., Lei, H., Hu, X., and Dong, W. (2020a). Hesperetin Ameliorates DSSInduced Colitis by Maintaining the Epithelial Barrier via Blocking RIPK3/ MLKL Necroptosis Signaling. Eur. J. Pharmacol. 873, 172992. doi:10.1016/j. ejphar.2020.172992
Zhang, X., Tang, N., Hadden, T. J., and Rishi, A. K. (2011). Akt, FoxO and Regulation of Apoptosis. Biochim. Biophys. Acta 1813 (11), 1978-1986. doi:10. 1016/j.bbamcr.2011.03.010

Zhang, Y., Han, D., Yu, S., An, C., Liu, X., Zhong, H., et al. (2020b). Protective Effect of Iridoid Glycosides of the Leaves of Syringa Oblata Lindl. On Dextran Sulfate Sodium-Induced Ulcerative Colitis by Inhibition of the TLR2/4/MyD88/ NF-kappaB Signaling Pathway. Biomed. Res. Int. 2020, 7650123. doi:10.1155/ 2020/7650123

Zhang, Y., Zha, Z., Shen, W., Li, D., Kang, N., Chen, Z., et al. (2021). Anemoside B4 Ameliorates TNBS-Induced Colitis through S100A9/MAPK/NF-kappaB Signaling Pathway. Chin. Med. 16 (1), 11. doi:10.1186/s13020-020-00410-1

Zhao, H. M., Huang, X. Y., Zhou, F., Tong, W. T., Wan, P. T., Huang, M. F., et al. (20132013). Si Shen Wan Inhibits mRNA Expression of Apoptosis-Related Molecules in P38 MAPK Signal Pathway in Mice with Colitis. Evid. Based Complement. Alternat Med., 432097. doi:10.1155/2013/432097

Zhu, H., Tong, S., Cui, Y., Wang, X., and Wang, M. (2021). Tanshinol Alleviates Ulcerative Colitis by Promoting the Expression of VLDLR. Drug Dev. Res.. doi: $10.1002 /$ ddr. 21840

Zhu, L., Dai, L. M., Shen, H., Gu, P. Q., Zheng, K., Liu, Y. J., et al. (2019a). Qing Chang Hua Shi Granule Ameliorate Inflammation in Experimental Rats and Cell Model of Ulcerative Colitis through MEK/ERK Signaling Pathway. Biomed. Pharmacother. 116, 108967. doi:10.1016/j.biopha.2019.108967

Zhu, L., Gu, P., and Shen, H. (2019b). Gallic Acid Improved Inflammation via NFkappaB Pathway in TNBS-Induced Ulcerative Colitis. Int. Immunopharmacol 67, 129-137. doi:10.1016/j.intimp.2018.11.049

Zhu, L., Shen, H., Zhang, L., and al, e. (2017). Effects of Baicalin on the Inflammation and Apoptosis in Ulcerative Colitis Rats Relating to PI3K/ AKT Pathway (China). China J. Traditional Chin. Med. Pharm. 32 (9), 4001-4004.

Conflict of Interest: The authors declare that the research was conducted in the absence of any commercial or financial relationships that could be construed as a potential conflict of interest.

Publisher's Note: All claims expressed in this article are solely those of the authors and do not necessarily represent those of their affiliated organizations, or those of the publisher, the editors and the reviewers. Any product that may be evaluated in this article, or claim that may be made by its manufacturer, is not guaranteed or endorsed by the publisher.

Copyright (C) $2022 \mathrm{Liu}$, Zeng, Wen, Huang and Liu. This is an open-access article distributed under the terms of the Creative Commons Attribution License (CC BY). The use, distribution or reproduction in other forums is permitted, provided the original author(s) and the copyright owner(s) are credited and that the original publication in this journal is cited, in accordance with accepted academic practice. No use, distribution or reproduction is permitted which does not comply with these terms. 